\title{
Probabilistic Entropy EMD Thresholding for Periodic Fault Signal Enhancement in Rotating Machine
}

\author{
Jian Dang, ${ }^{1,2}$ Rong Jia, ${ }^{1,2}$ Hua Wu, ${ }^{2}$ Xingqi Luo,, ${ }^{1,2}$ and Diyi Chen ${ }^{3}$ \\ ${ }^{1}$ State Key Laboratory Base of Eco-Hydraulic Engineering in Arid Area, Xian University of Technology, Xian 710048, China \\ ${ }^{2}$ Institute of Water Resources and Hydro-Electric Engineering, Xian University of Technology, Xian 710048, China \\ ${ }^{3}$ Institute of Water Resources and Hydropower Research, Northwest A\&F University, Yangling 712100, China
}

Correspondence should be addressed to Rong Jia; jiarong@xaut.edu.cn

Received 1 March 2017; Accepted 20 June 2017; Published 30 July 2017

Academic Editor: Marc Thomas

Copyright (C) 2017 Jian Dang et al. This is an open access article distributed under the Creative Commons Attribution License, which permits unrestricted use, distribution, and reproduction in any medium, provided the original work is properly cited.

\begin{abstract}
Since the slight fault feature of incipient fault is usually polluted by heavy background noise, it is difficult to extract the weak feature signal in rotating machine. As an adaptive decomposing technique, empirical mode decomposition (EMD) based denoising methods have a good effect on the feature separation and noise elimination. However, for rotating machine with poor working environment, the components attributed to noise might have higher amplitudes, which restrict the efficiency of noise reduction in current EMD-based denoising methods. Therefore, a probabilistic entropy EMD thresholding algorithm for periodic fault signal enhancement in rotating machine is proposed in this paper. In this method, the entropy threshold of each IMF is constructed instead of the threshold applied to $N$ sampling points of each IMF directly, which overcomes the shortcoming of the denoising effect limited by larger amplitude noise reservation and smaller amplitude feature signal reduction in the current denoising methods. Meanwhile, in order to make the amplitudes of all the IMF reduce in a smooth way, a multiscale thresholding algorithm based on quantile statistics to provide probability indexes is presented. Engineering application demonstrates that the proposed method is effective in the noise reduction and fault feature enhancement in the rotating machine.
\end{abstract}

\section{Introduction}

With the rapid development of science and technology, rotating machine plays a significant role in a wide range of industrial applications, such as hydroelectric turbine, wind turbine, aeroengine, transportation vehicles, and machine tools. Hence, it is of great significance to research feature extraction methods for incipient fault prognosis and guaranteeing the reliability of the mechanical system [1]. In the past decades, many algorithms are proposed in the area of mechanical intelligent diagnosis [2-4]. However, as researchers found that the rotating machine is a complicated and nonlinear system, it has been proven that the slight fault feature is usually overwhelmed by heavy background noise, which makes it difficult to detect the weak feature in the early failure of the rotating machine.

Empirical mode decomposition (EMD) was first introduced by Huang et al. in 1998 [5]. As a powerful adaptive decomposition tool, EMD can break the signal down into a number of amplitude and frequency modulated (AM/FM) zero-mean signals, termed intrinsic mode functions (IMFs), and has been widely used to analyze the nonstationary and nonlinear signal processes [6-8].

Recently, based on the statistical characteristics analysis of white Gaussian noise and fractional Gaussian noise in EMD sifting process [9-11], Flandrin et al. put forward an EMD denoising scheme with partial reconstruction (EMD-PR) of relevant IMFs in an adaptive way [12], and many attempts have been made to select relevant IMFs in an efficient way [13-20]. Boudraa and Cexus proposed a distortion measure method called consecutive mean square error (CMSE) to determine the relevant IMFs [13]. Ricci and Pennacchi [14] and Albert and Nii [15] developed a merit index and correlation coefficient to realize the automatic selection of relevant IMFs, respectively. Komaty et al. [20] provided a new EMDbased filtering algorithm, in which the relevant IMFs were selected on the basis of a striking similarity between the 
probability density function of the measured signals and each IMF.

However, for the weak feature signal in the early failure of rotating machine, it is difficult to distinguish the noise components and feature signal by selecting the relevant IMFs and it is disastrous for noise removal when the selection is incorrect.

In [21], Kopsinis and McLaughlin proposed an alternative EMD-based denoising procedure inspired by the wavelet thresholding principle [22], which included the direct EMD thresholding (EMD-DT) and EMD interval thresholding (EMD-IT). However, in contrast to wavelet-based denoising method where the threshold is applied on the wavelet coefficients, the IMF thresholding is directly applied to timedomain signals of each IMF in EMD-based denoising procedure $[23,24]$. Due to the fact that the periodic fault signal usually submerges in the strong background noise, the larger amplitude noise will be kept and smaller amplitude feature signal will be discarded in the traditional EMD thresholding methods.

According to the above-mentioned shortcomings, a probabilistic entropy EMD thresholding algorithm is proposed in this paper for periodic fault signal enhancement of rotating machine. Permutation entropy [25], as a statistical measurement method, has high sensibility to abrupt dynamic change of time series, which is prone to discriminate the feature signal from the noise. Fu-zhou et al. introduced permutation entropy to distinguish the working condition of rolling bearing and proved the validity of permutation entropy in online abnormality detection [26]. Motivated by this advantage, this paper investigates the utility of permutation entropy to separate the noise from the feature signal efficiently in each IMF and the entropy threshold of each IMF is constructed in the proposed method instead of the threshold applied to $N$ sampling points of each IMF directly, which overcomes the problems of larger amplitude noise reservation and smaller amplitude feature signal reduction in the traditional EMD-based denoising method. In addition, to make the amplitudes of all the IMF reduce in a smooth way, a multiscale thresholding algorithm based on quantile statistics to provide probability indexes is presented for the periodic fault signal enhancement. Engineering application demonstrates that the proposed method is effective in the noise reduction and periodic fault signal enhancement in rotating machine.

This paper is organized as follows. The EMD-based denoising methods are introduced in Section 2, which includes EMD-based partial reconstruction and IMF thresholding-based denoising methods. Section 3 describes the proposed probabilistic entropy EMD thresholding algorithm. Then, the effectiveness of the proposed method is verified by the engineering application in Section 4. Finally, conclusions are drawn in Section 5.

\section{EMD-Based Denoising Method}

2.1. Brief Review of EMD. As an innovative time series analysis tool developed by Huang et al., EMD [5] adaptively decomposes multicomponent signals $x(t)$ into a number $L$ of the so-called IMFs $h^{(i)}(t)$. The IMF should satisfy the following two conditions: (1) in the whole data, the number of zero-crossings and extrema must either equal or differ at most by one; (2) at any point, the mean value of envelope defined by local maxima and the envelope defined by the local minima is zero. The procedures of EMD decomposition are shown as the following steps list below.

(1) Set $r_{o}(t)=x(t)$ and $i=1$.

(2) Identify all the local extrema of the signal being analyzed.

(3) Connect the local maxima and minima to construct the upper and lower envelopes by a cubic spline interpolation.

(4) Compute the mean $m_{i-1}(t)$ of upper and lower envelopes.

(5) Extract the local oscillation mode: $\operatorname{IMF}(i)=r_{i-1}(t)-$ $m_{i-1}(t)$. If $\operatorname{IMF}(i)$ satisfies the two conditions of IMF, it is treated as an IMF and let $h^{(i)}(t)=\operatorname{IMF}(i)$. Otherwise take $\operatorname{IMF}(i)$ as the original signal and repeat steps (1)-(3) until $\operatorname{IMF}(i)$ satisfies the two conditions.

(6) Define remainder $r_{i}(t)=r_{i-1}(t)-h^{(i)}(t)$. If $r_{i}(t)$ still has at least 2 extrema, go to step (2) with $i=i+1$. Then, the original signal can be reached by the sum of IMFs and residue described as follows:

$$
x(t)=\sum_{i=1}^{L} h^{(i)}(t)+r_{L}(t),
$$

where $r_{L}(t)$ is the residual that is a nonzero-mean slowly varying function with only few extrema and $L$ is the number of IMFs. The time-domain waveform and frequency spectrum of simulation signal $x(t)$ are illustrated in Figure 1.

$$
\begin{aligned}
& x(t)=s_{1}(t)+s_{2}(t)+d_{1}(t)+H(t) * d_{2}(t) \\
& s_{1}(t)=\sin \left(2 \pi f_{1} t\right) f_{1}=3 \mathrm{~Hz} \\
& s_{2}(t)=\sin \left(2 \pi f_{2} t\right) f_{2}=5 \mathrm{~Hz} \\
& d_{1}(t)=\sqrt{2 D_{1}} \varepsilon(t) D_{1}=0.5 \\
& d_{2}(t)=\sqrt{2 D_{2}} \varepsilon(t) D_{2}=2,
\end{aligned}
$$

where $H(t)$ is a step function, $H(t)=0, t \in(0,485) \cup$ $(505,990) \cup(1010,1495) \cup(1515,2000]$, and $H(t)=1, t \in$ $[485,505] \cup[990,1010] \cup[1495,1515]$.

As shown in (2), the simulation signal $x(t)$ is composed by multiple signals with different frequency, white Gaussian noise, and impact noise. However, it can be observed from Figure 2 that the noise signals have been decomposed to each IMF in the sifting process of EMD and the original sinusoidal signal cannot be obtained from the EMD result.

2.2. EMD-Based Partial Reconstruction. As a conventional denoising method, the EMD-based partial reconstruction is used to check whether a specific IMF contains useful information or primarily noise. To analyze the decomposition effect of white Gaussian noise, the noise-only IMF energies can be described as follows [12]:

$$
E_{k}=E_{1}^{2} \beta^{-1} \rho^{-k}, \quad k=2,3,4, \ldots,
$$



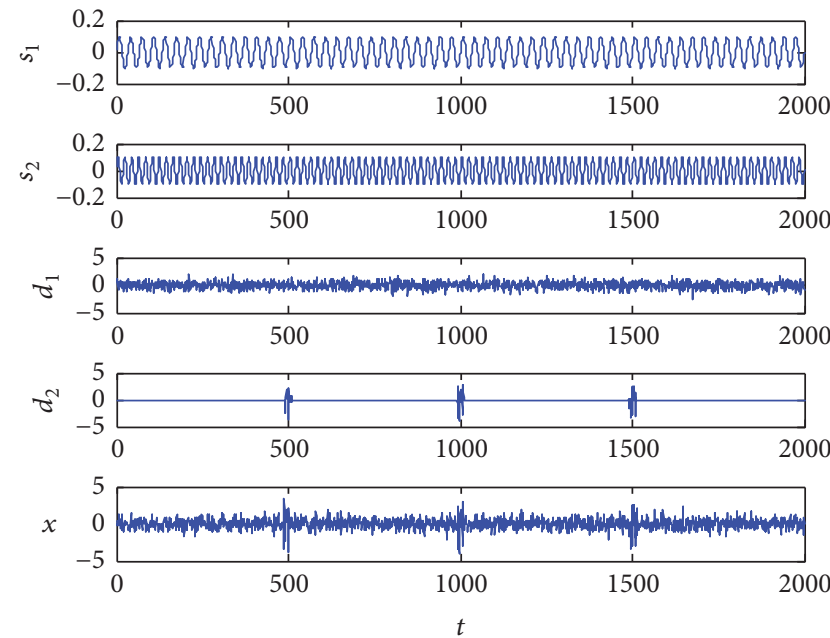

(a)

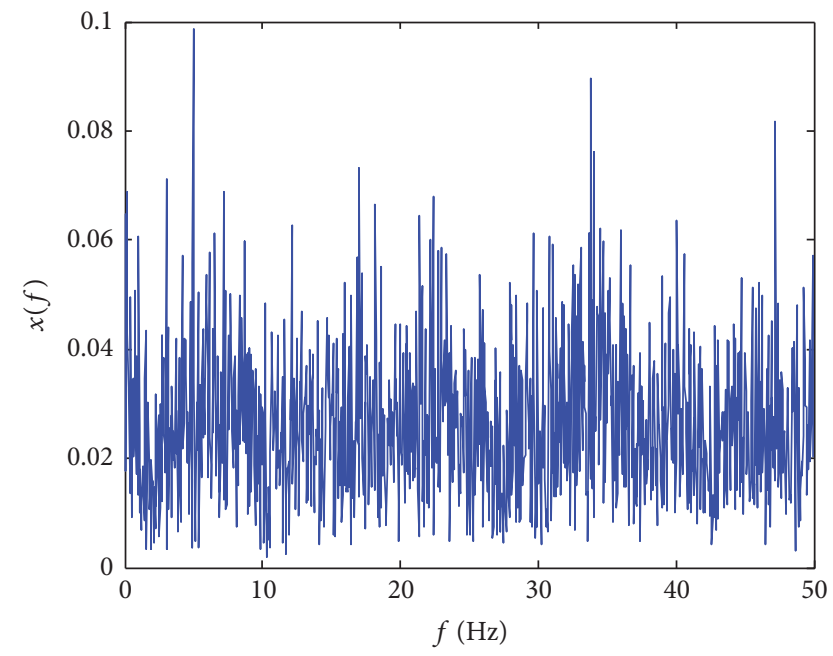

(b)

FIGURE 1: The noisy signal $x(t)$ : (a) time-domain waveform, (b) frequency spectrum.
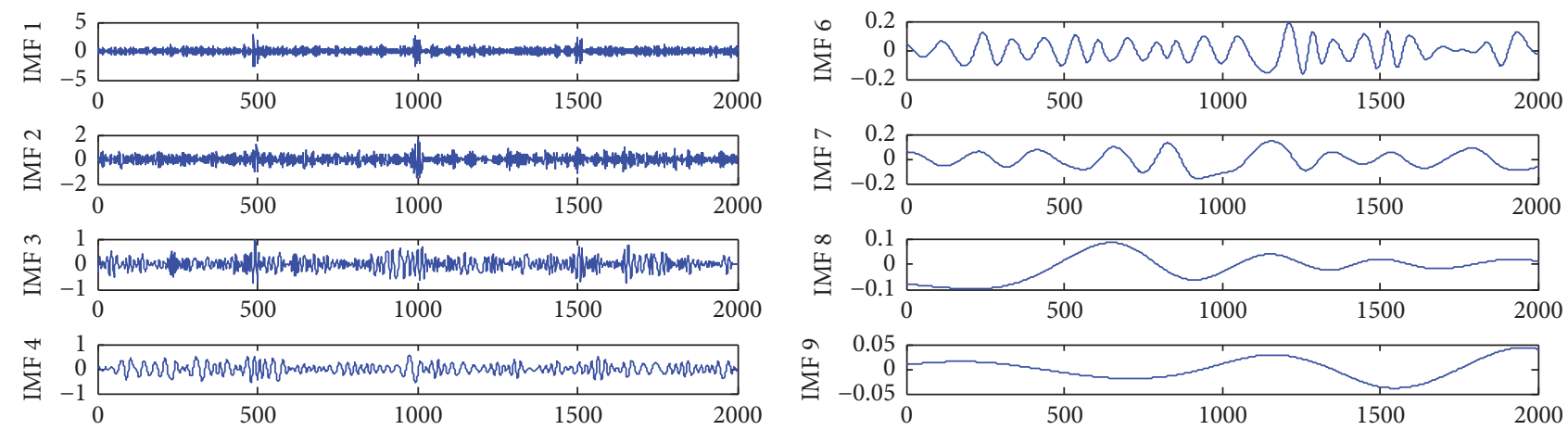

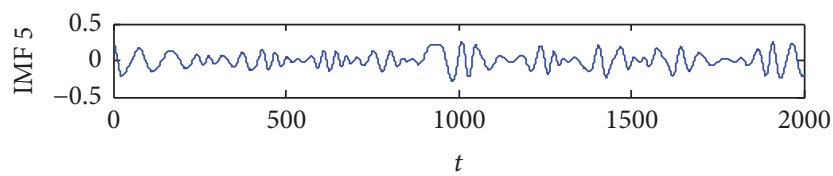

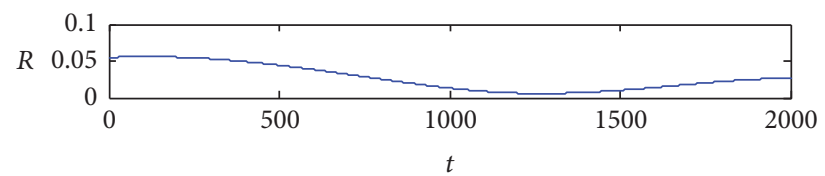

Figure 2: Empirical mode decomposition of the noisy signal $x(t)$.

where Flandrin et al. [12] have proposed the value 0.719 and 2.01 for parameters $\rho$ and $\beta$, respectively, based on the estimation by a large number of independent noise realizations and IMFs, and $E_{k}$ is the energy of $k$ th IMF.

Figure 3 shows the energy variation curve of IMFs originated from the EMD sifting process of the noisy signal $x(t)$ in Figure 1(a). It can be seen that, after the third IMF, the actual energies have a significant change compared with the estimated energies of the noise-only IMFs, which indicates the appearance of feature signal. Therefore, the partial signal reconstruction including IMFs numbers 4 to 9 results in the denoised signals. However, based on the observation of denoising results in Figure 4, the sinusoidal signal with $5 \mathrm{~Hz}$ is discarded in the EMD-PR denoising results. Actually, it is difficult to separate the noise component and feature signal by selecting the relevant IMFs in heavy noise background.

2.3. IMF Thresholding-Based Denoising. Considering the problem of feature signal missing in EMD-PR denoising method, an alternative EMD-based denoising procedure in each IMF enlightened by the wavelet thresholding is proposed and developed. The IMF thresholding-based denoising methods can be grossly divided into EMD-DT and EMD-IT [22]. The EMD-DT for hard thresholding can be described as

$$
H^{(i)}(t)= \begin{cases}0 & \left|h^{(i)}(t)\right|<T_{i} \\ h^{(i)}(t) & \left|h^{(i)}(t)\right|>T_{i}\end{cases}
$$

and that for soft thresholding [27] can be described as

$$
H^{(i)}(t)= \begin{cases}0 & \left|h^{(i)}(t)\right|<T_{i} \\ \operatorname{sgn}\left(h^{(i)}(t)\right)\left(h^{(i)}(t)-T_{i}\right) & \left|h^{(i)}(t)\right|>T_{i},\end{cases}
$$

where $H^{(i)}(t)$ indicates the $i$ th thresholded IMF and $T_{i}$ represents the threshold of the $i$ th IMF. Then, according to the universal threshold $T=\sigma \sqrt{2 \ln N}$, multiples of the IMF 


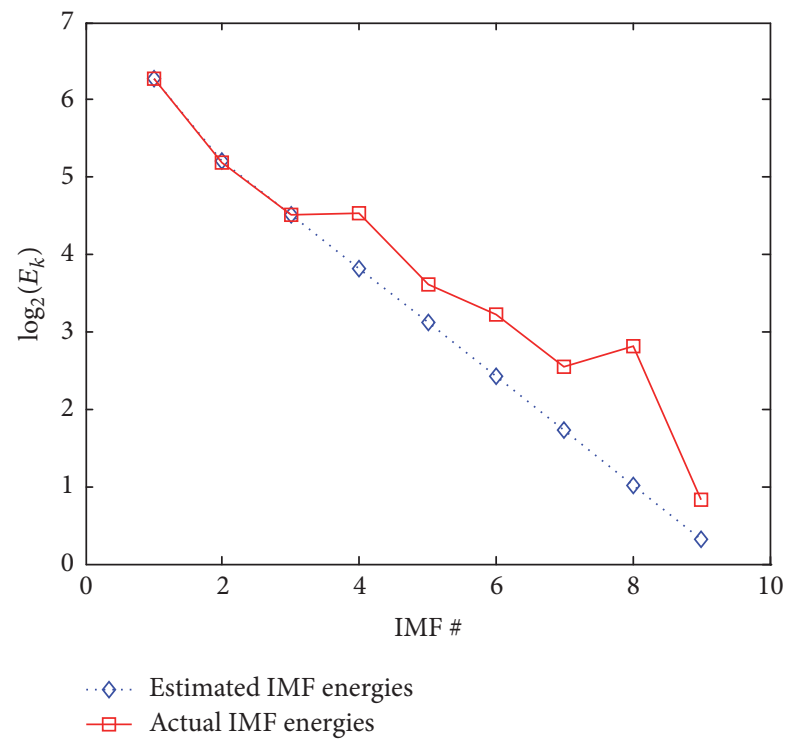

FIGURE 3: Difference between theoretical noise-only energies and actual IMF energies with respect to IMF number.

threshold are proposed as $T_{i}=C \sqrt{2 E_{i} \ln N}$ for the EMD thresholding methods, where $C$ is a constant and $E_{i}$ is the energy of the $i$ th IMF.

Nevertheless, it has been proven that the EMD-DT can result in disastrous consequence for the continuity of the reconstructed signal. Therefore, based on the above analysis, Donoho and Johnstone [22] put forward an EMD-IT method to reduce the discontinuity of denoised signals. In any interval between the two adjacent zero-crossings $Z_{j}^{(i)}=\left[z_{j}^{(i)} z_{j+1}^{(i)}\right]$, the signal of $i$ th IMF is defined as a basic analyzable mode cell; the introduction of the EMD-IT for hard thresholding is shown in

$$
H^{(i)}\left(\left(Z_{j}^{(i)}\right)\right)= \begin{cases}0 & \left|h^{(i)}\left(r_{j}^{(i)}\right)\right|<T_{i} \\ h^{(i)}\left(\left(Z_{j}^{(i)}\right)\right) & \left|h^{(i)}\left(r_{j}^{(i)}\right)\right|>T_{i}\end{cases}
$$

and that for soft thresholding [27] is shown in

$$
\begin{aligned}
& H^{(i)}\left(\left(Z_{j}^{(i)}\right)\right) \\
& = \begin{cases}0 & \left|h^{(i)}\left(r_{j}^{(i)}\right)\right|<T_{i} \\
h^{(i)}\left(\left(Z_{j}^{(i)}\right)\right) \frac{\left(h^{(i)}\left(\left(r_{j}^{(i)}\right)\right)-T_{i}\right)}{h^{(i)}\left(\left(r_{j}^{(i)}\right)\right)} & \left|h^{(i)}\left(r_{j}^{(i)}\right)\right|>T_{i},\end{cases}
\end{aligned}
$$

where $H^{(i)}\left(\left(Z_{j}^{(i)}\right)\right)$ indicates the sampling points from instants $z_{j}^{(i)}$ to $z_{j+1}^{(i)}$ of the $i$ th IMF. In order to illustrate the difference between the EMD-DT and EMD-IT scheme, the third IMF of the simulation signal $x(t)$ is used as an example which is shown in Figure 5. In addition, Figures 6 and 7 show the denoising results of the noisy signal $x(t)$ with EMD-DT and EMD-IT method, respectively.

However, from the observation of Figures 6(a) and 7(a), since the scheme of current EMD-based denoising methods including EMD-PR, EMD-DT, and EMD-IT is that the threshold is applied to $N$ sampling points of each IMF directly, the impact noise signal with larger amplitude is not eliminated by IMF threshold-based denoising methods.

\section{Proposed Probabilistic Entropy EMD Thresholding (EMD-PE)}

Based on the above problems and analysis, the entropy threshold is introduced instead of the threshold applied to $N$ sampling points of each IMF directly, and a multiscale thresholding algorithm based on quantile statistics for providing probability indexes is proposed in this paper. As a measure of signal complexity, permutation entropy (PE) [25] has the characteristics of high sensitivity to abrupt signal change and simple calculation and strong robustness [28], and it has been widely applied to research the complexity of the time series and dynamic characteristics $[29,30]$.

To reflect the signal change in real time, the sliding window is constructed in each IMF, where the width of sliding window can be selected according to the fault characteristic frequency information [31] and the sliding distance is 1 . The original signal can be segmented into multiple subsignals by shifting the constructed sliding window. Figure 8 displays the diagrammatic sketch of the constructed sliding window.

The proposed EMD-PE algorithm translates to

$$
H^{(i)}(t)= \begin{cases}0 & \left|H_{p}^{(i)}(t)\right|>T_{i} \\ h^{(i)}(t) * \operatorname{Prob}^{(i)}(t) & \left|H_{p}^{(i)}(t)\right|<T_{i},\end{cases}
$$

where $H_{p}^{(i)}(t)$ is the PE of the $i$ th IMF and $\operatorname{Prob}^{(i)}(t)$ denotes the probability index of $i$ th IMF, which represents the original signal's likelihood of being periodic fault signal.

$$
\begin{aligned}
& \operatorname{Prob}^{(i)}(t)= \begin{cases}0 & i=1 \\
\frac{1}{M} \sum_{j=1}^{M} \mathrm{PI}_{(j)}^{(i)}(t) * 100 \% & i=2,3, \ldots, L,\end{cases} \\
& \mathrm{PI}_{(j)}^{(i)}(t)= \begin{cases}1 & H_{p}^{(i)}(t)<\mathrm{Qua}\left[H_{p}^{(i)}(t), \alpha_{i j}\right] \\
0 & H_{p}^{(i)}(t)>\operatorname{Qua}\left[H_{p}^{(i)}(t), \alpha_{i j}\right]\end{cases} \\
& \alpha_{i j}=j * \frac{E_{1}^{*}-E_{i}^{*}}{M * E_{1}^{*}},
\end{aligned}
$$

where Qua $\left[H_{p}^{(i)}(t), \alpha_{i j}\right]$ indicates the $\alpha_{i j}$ th quartile of $H_{p}^{(i)}(t)$, $M$ represents the number of multiscale thresholds at each IMF, and $E_{1}^{*}$ and $E_{i}^{*}$ are the energies of first IMF and $i$ th IMF, respectively. Inspired by the IMF-dependent universal threshold proposed by [20], the baseline entropy threshold in each IMF is defined in

$$
T_{i}= \begin{cases}\min \left(H_{p}^{(i)}(t)\right) & i=1 \\ \text { Qua }\left[H_{p}^{(i)}(t), \frac{E_{1}^{*}-E_{i}^{*}}{E_{1}^{*}}\right] & i=2,3, \ldots, L .\end{cases}
$$

The result is illustrated by the example of third IMF of the simulation signal $x(t)$. Figure 9 shows the variation curve of 


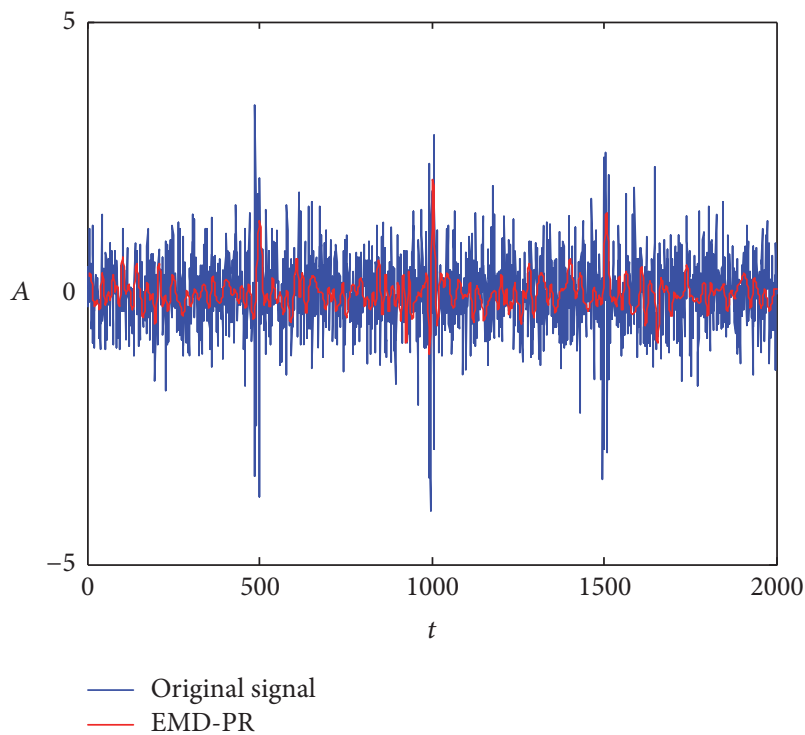

(a)

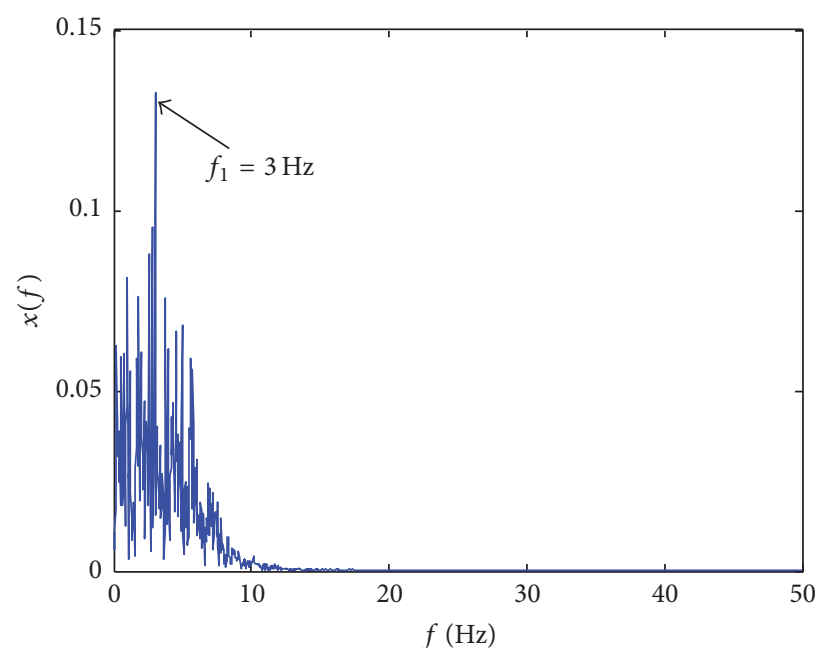

(b)

FIGURE 4: EMD-PR denoising results of the noisy signal $x(t)$ : (a) time-domain waveform, (b) frequency spectrum.

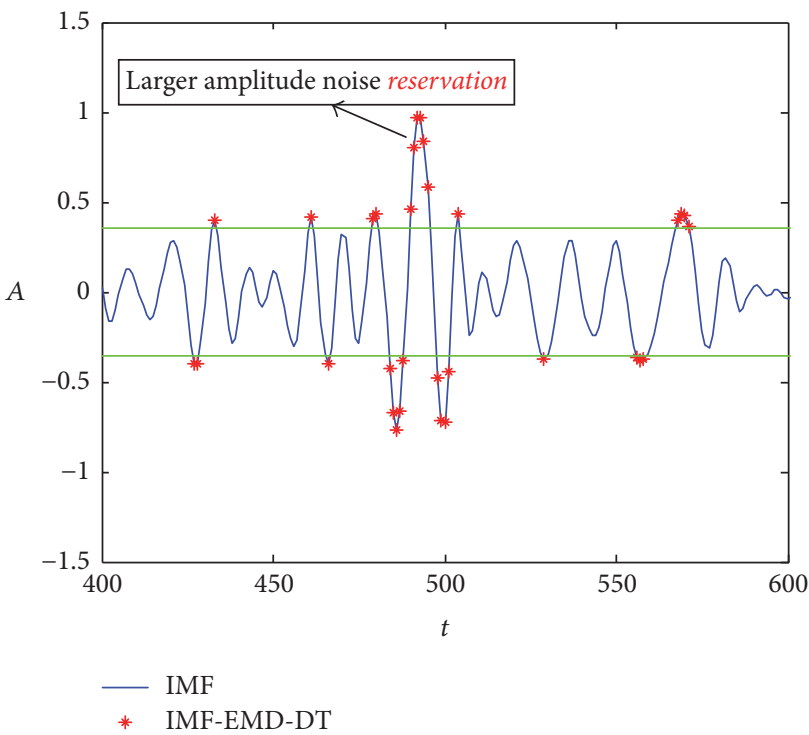

(a)

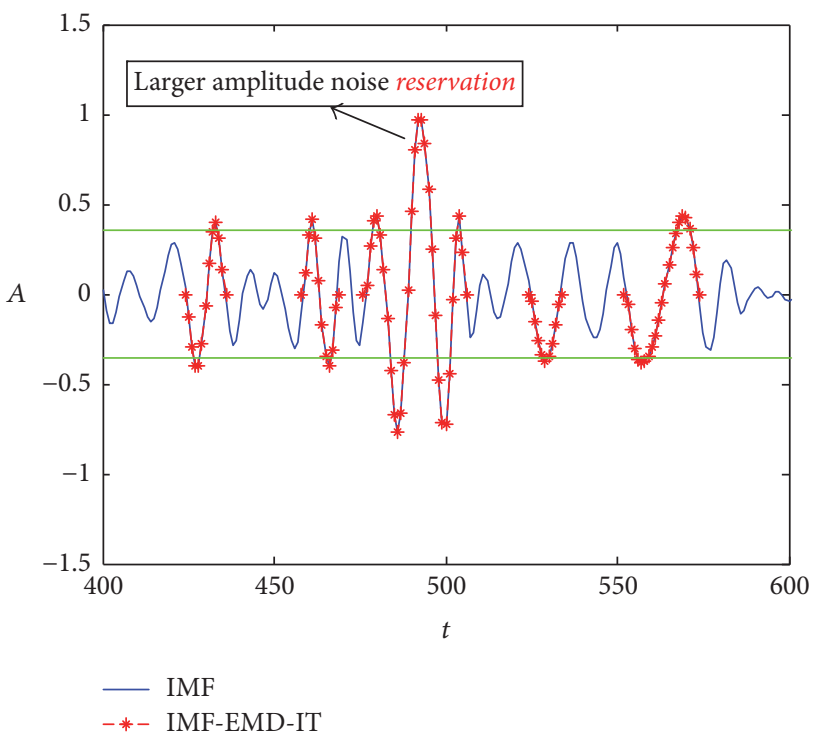

(b)

FIGURE 5: Difference between the schemes of EMD-DT and EMD-IT: (a) EMD-DT, (b) EMD-IT.

PE value of the third IMF. It can be seen that the value $H_{p}$ has a significant increase in the case where the amplitude of noise signal is relatively large, which indicates that the PE value of each IMF can be settled as an index to separate the noise from the feature signal.

In addition, the quantile statistics approach is provided in the construction of multiscale thresholds in the proposed EMD-PE algorithm. Figure 10(a) depicts the cumulative distribution function (CDF) of PE values of the third IMF, where $\lambda_{j}$ denotes the $\alpha_{i j}$ quantile of PE. Meanwhile, based on the introduction of the idea of quantile statistics, the probability indexes are calculated to make the amplitude of each IMF reduce in a smooth way, which is illustrated in Figure 10(b). Figure 11 shows the denoising results of the noisy signal $x(t)$ with the proposed EMD-PE method. By comparison with the effects in Figures 6 and 7, the proposed method in this paper can effectively solve the larger amplitude noise keeping problem in the traditional EMDbased denoising methods.

A thorough denoising performance evaluation of the proposed EMD-PE method and current EMD-based denoising methods is realized using the SNR and root mean square error (RMSE), as illustrated in Figure 12. The SNR of the original simulation signal constructed in (2) is -16.59 . Evidently, 


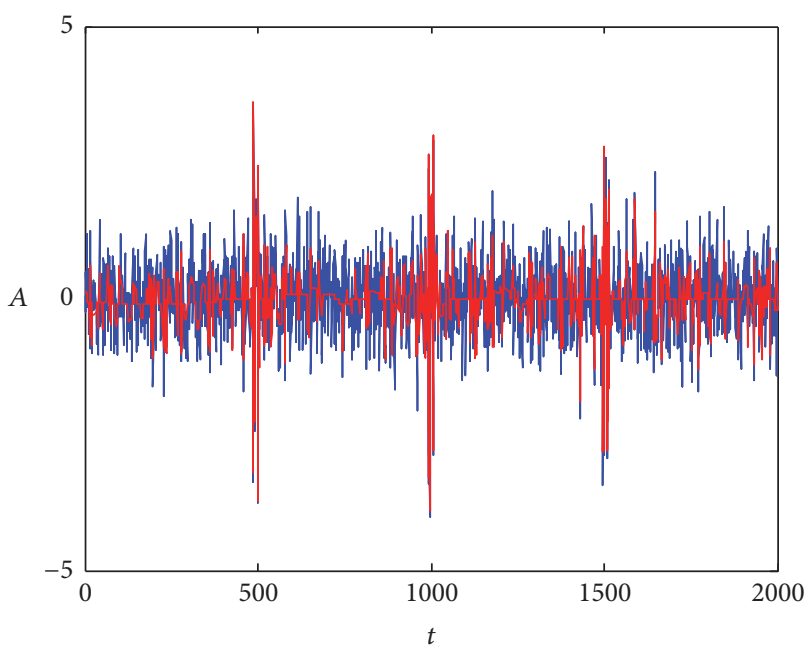

- Original signal
EMD-DT

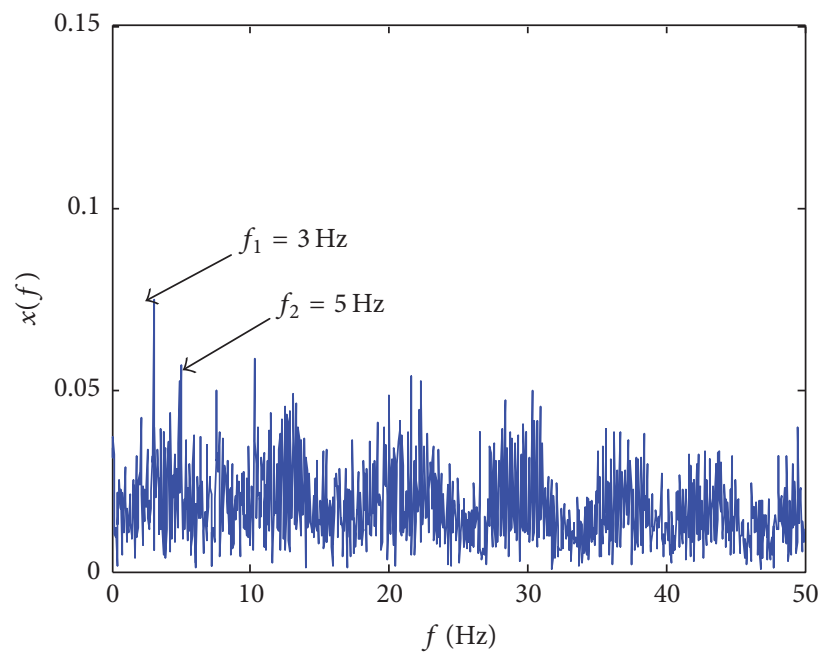

(b)

FIGURE 6: EMD-DT denoising results of the noisy signal $x(t)$ : (a) time-domain waveform, (b) frequency spectrum.

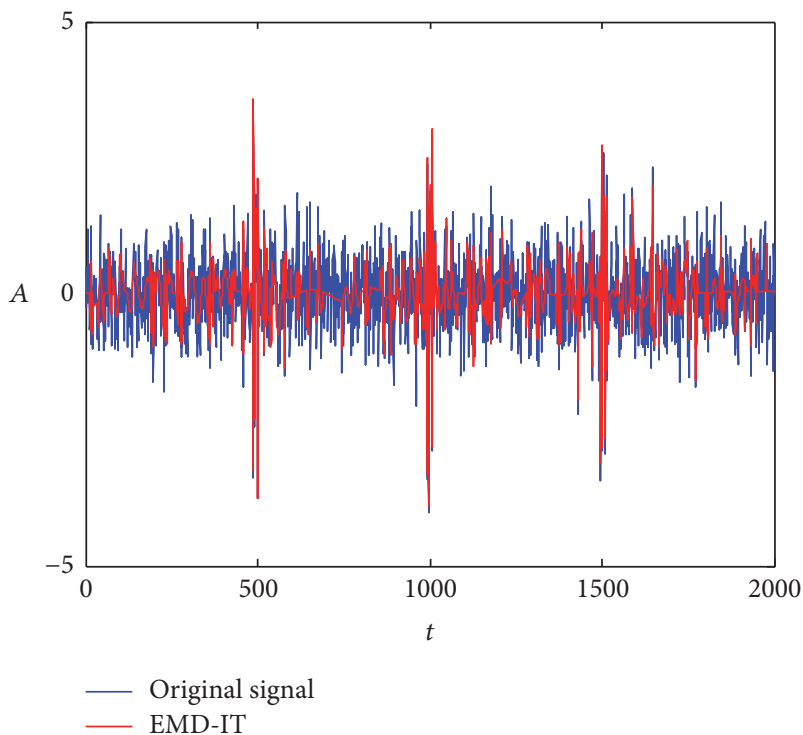

(a)

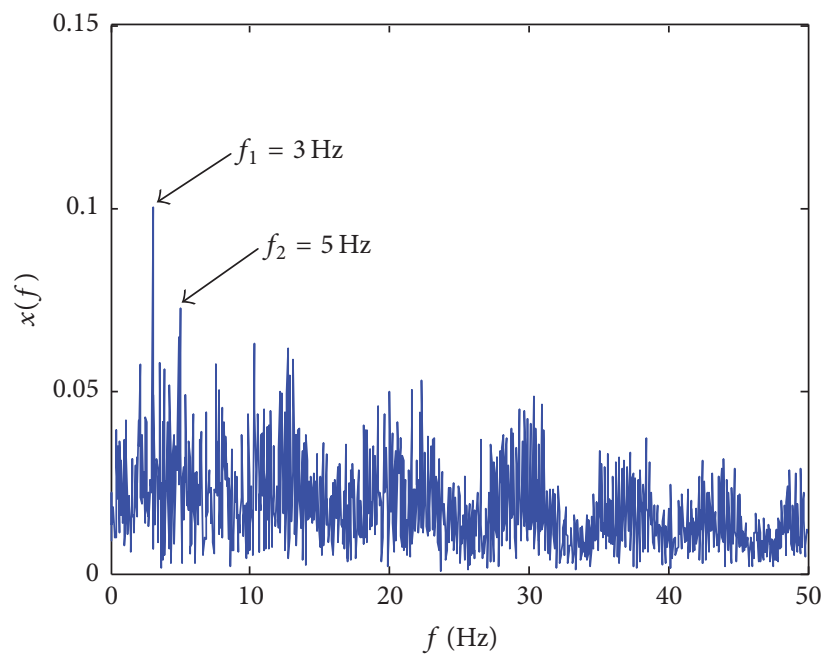

(b)

FIGURE 7: EMD-IT denoising results of the noisy signal $x(t)$ : (a) time-domain waveform, (b) frequency spectrum.

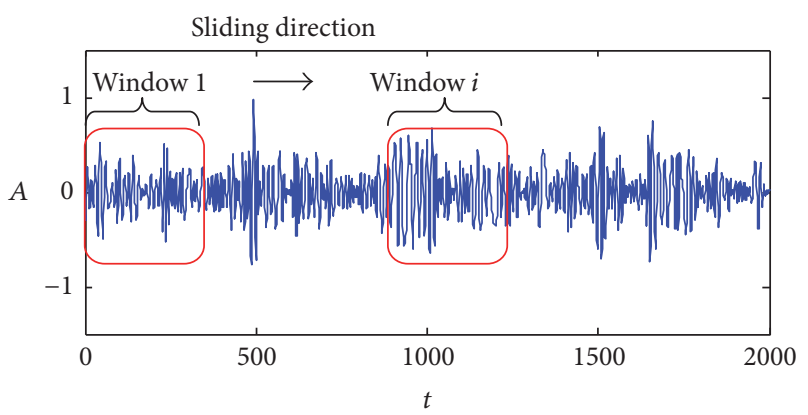

FIGURE 8: Diagrammatic sketch of constructed sliding window.

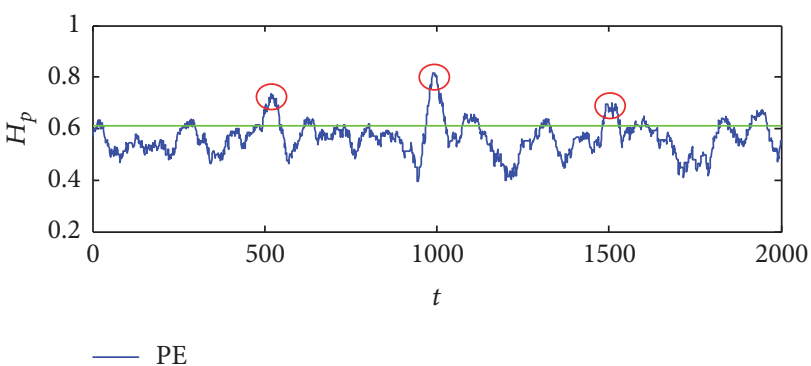

FIGURE 9: Variation curve of PE value of the third IMF of noisy signal $x(t)$. 


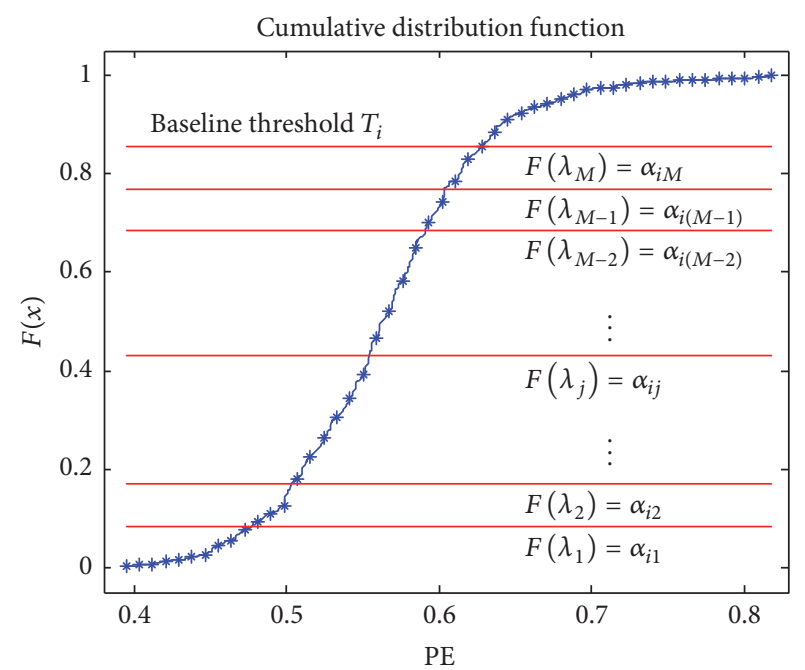

(a)

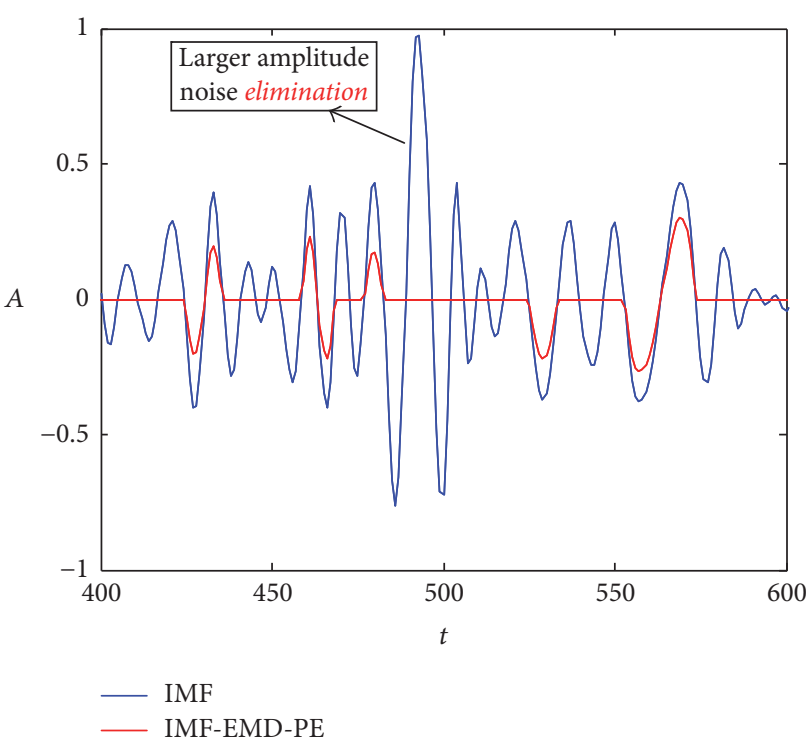

(b)

FIgure 10: The scheme of proposed EMD-PE algorithm: (a) the CDF of PE values, (b) EMD-PE.

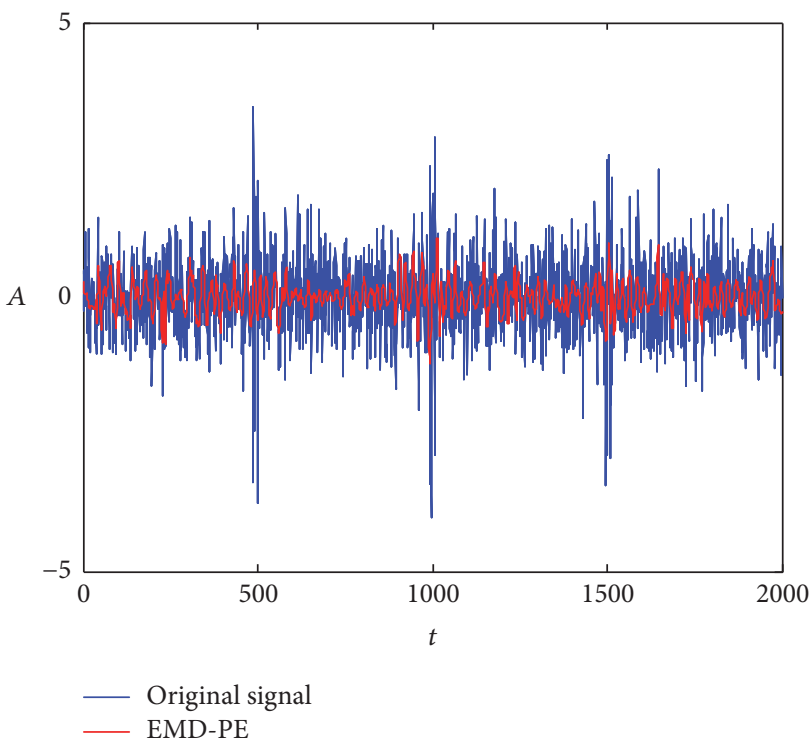

(a)

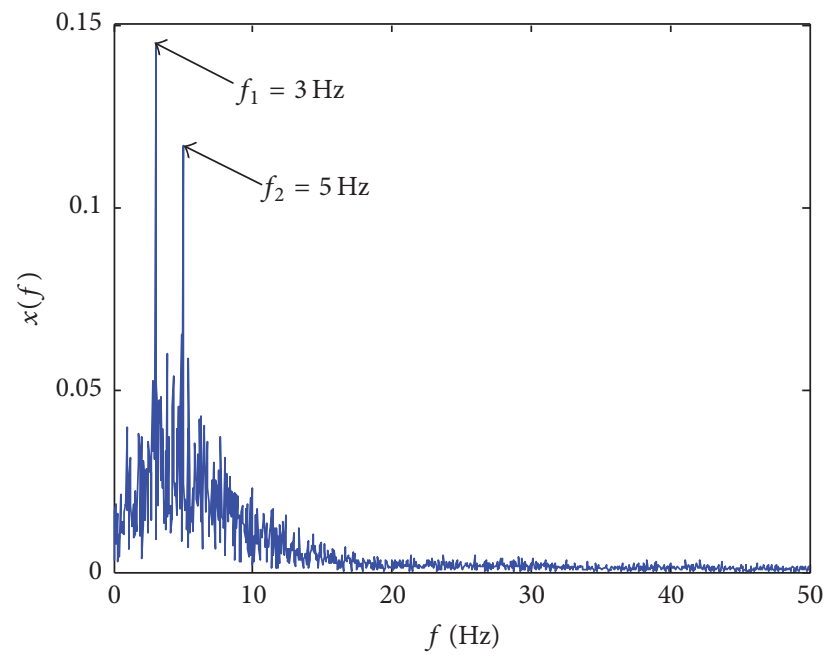

(b)

FIGURE 11: EMD-PE denoising results of the noisy signal $x(t)$ : (a) time-domain waveform, (b) frequency spectrum.

compared with the current EMD-based denoising methods, the proposed algorithm has a better effect on signal reduction and periodic fault signal enhancement.

\section{Engineering Application}

4.1. Experimental Data Collection. To verify the effectiveness of the proposed method in the fault feature extraction of rotating machine, the experimental studies on hydroelectric turbine in upper reaches of the Yellow River are conducted. As shown in Figure 13, the experimental signals are acquired from the prototype of hydroelectric turbine with 5 blades, and the rated speed is $107.1 \mathrm{r} / \mathrm{min}(1.79 \mathrm{~Hz})$. Figure 14 describes specific layout of measuring points of pressure fluctuation signals in the turbine.

In the hydroelectric turbine, since the oil supply is generally sustained by the floating tile, the floating tile is usually affected by vibration of the turbine shaft, which makes it prone to wear. In addition, once the floating tile suffers damage, the problems of oil leakage and its diffusion to other channels in the oil-supply will lead to the turbine being unfeasible to work in the on-cam operating condition. For these reasons, faults on the floating tile in the oil supply are created in the experiment. The vibration 


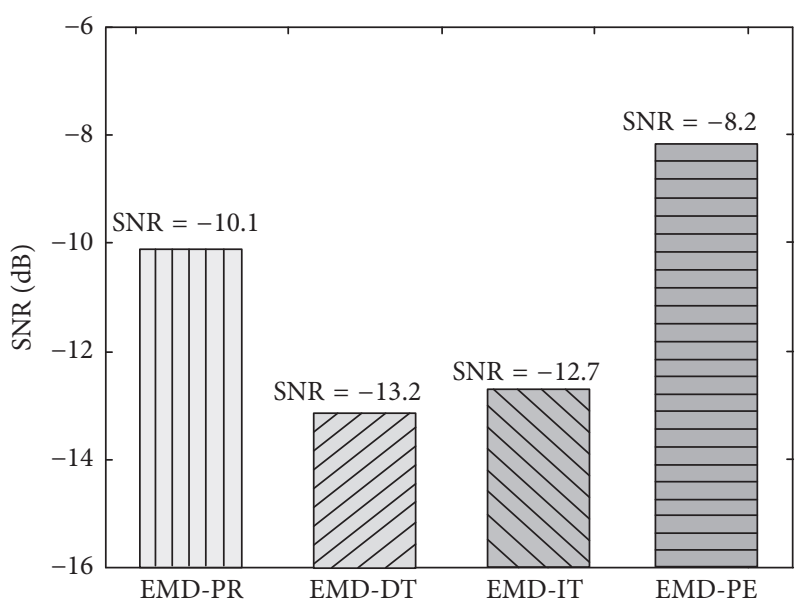

(a)

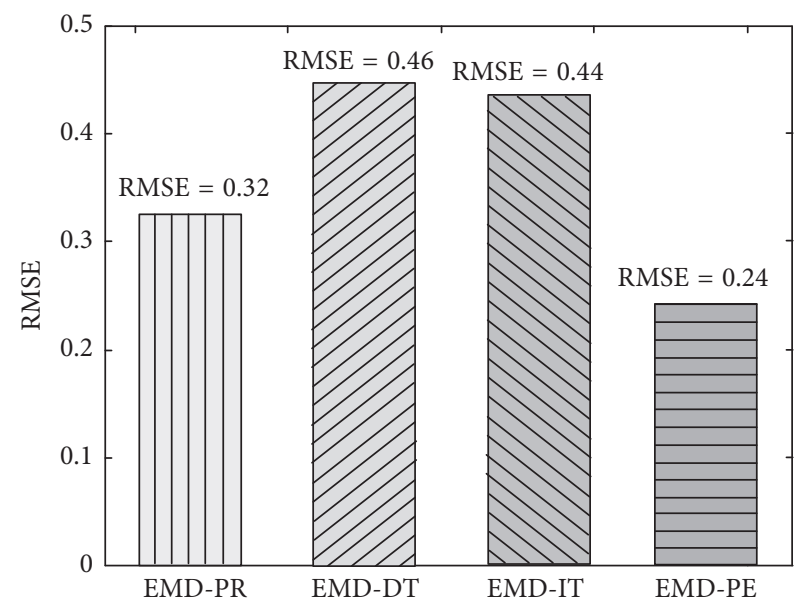

(b)

FIGURE 12: Performance evaluation of the proposed EMD-PE method and current EMD-based denoising methods: (a) SNR, (b) RMSE.

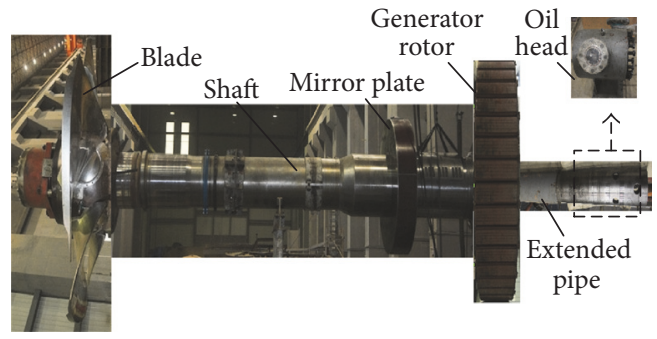

FIGURE 13: Prototype of hydroelectric turbine.

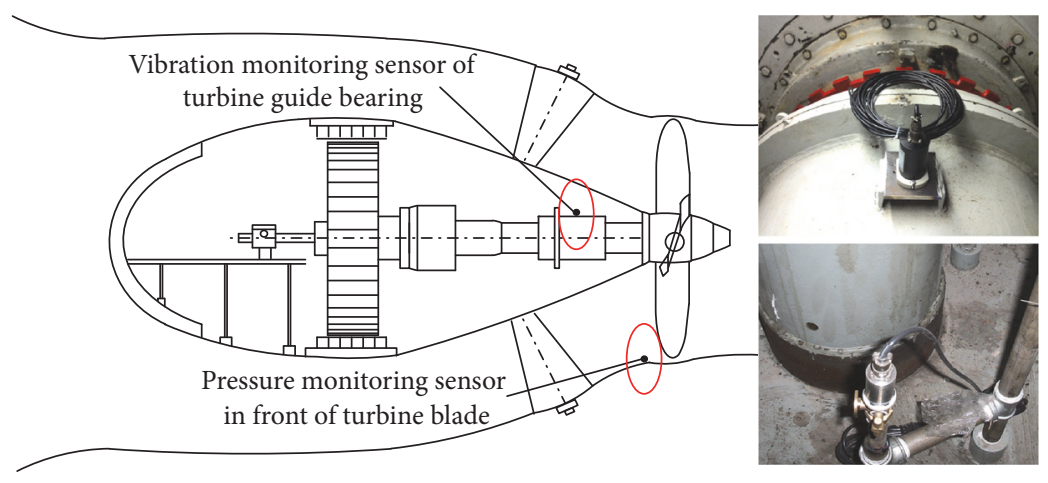

FIGURE 14: Specific layout of measuring points in hydroelectric turbine.

signals of turbine guide bearing and pressure fluctuation signals before the turbine blade are measured under the wear of floating tile, respectively, as shown in Figure 15. Each sampling length is 1000 points, and the sampling frequency is $227 \mathrm{~Hz}$.

4.2. Experimental Results under the Wear of Floating Tile. The proposed method is applied to periodic fault signal enhancement of rotating machine under the wear of floating tile in this section. Due to the fact that the experimental signals are acquired from the prototype of hydroelectric turbine with 5 blades and the rated speed is $107.1 \mathrm{r} / \mathrm{min}$ $(1.79 \mathrm{~Hz})$, the fault features of floating tile are the blade passing frequency of $8.95 \mathrm{~Hz}$ and its harmonic components, which is related to the impact signals of water on 5 runner blades. Figure 16 illustrates the time-domain waveform and frequency spectrum of vibration signals under the wear of floating tile. Obviously, apart from rotating frequency, the fault characteristic frequency $8.95 \mathrm{~Hz}$ signals are almost swamped by the heavy noise.

The experimental results of vibration signals under the wear of floating tile with EMD-PR, EMD-DT, EMD-IT, and proposed EMD-PE method are shown in Figures 17(a)-17(h), respectively. It can be seen from Figures 17 (a) and 17(b) that the fault characteristic signal has a serious attenuation although the EMD-PR method offers a better noise 

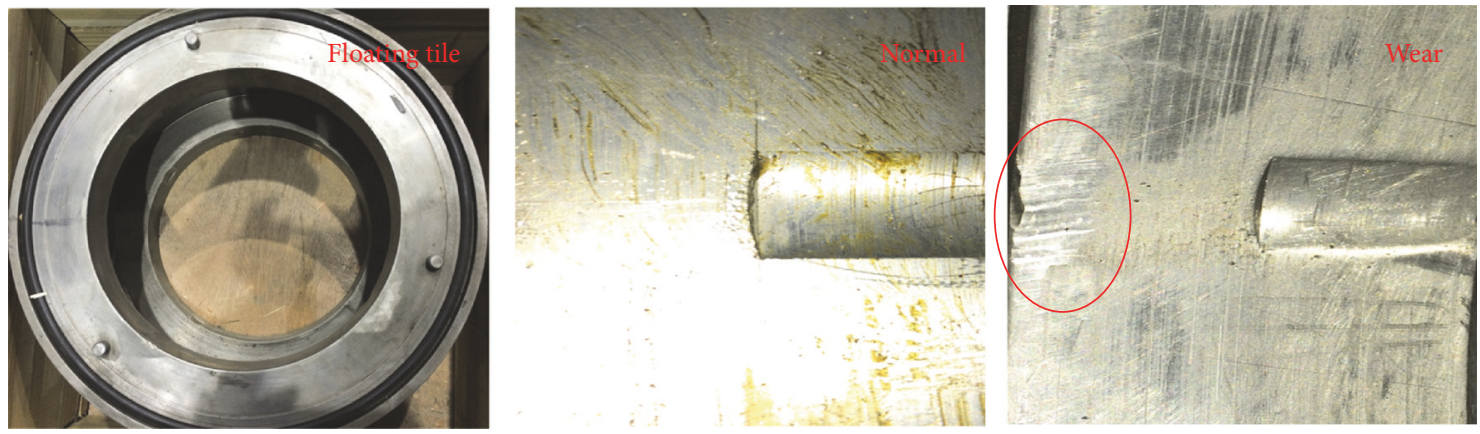

FIGURE 15: The wear of floating tile.

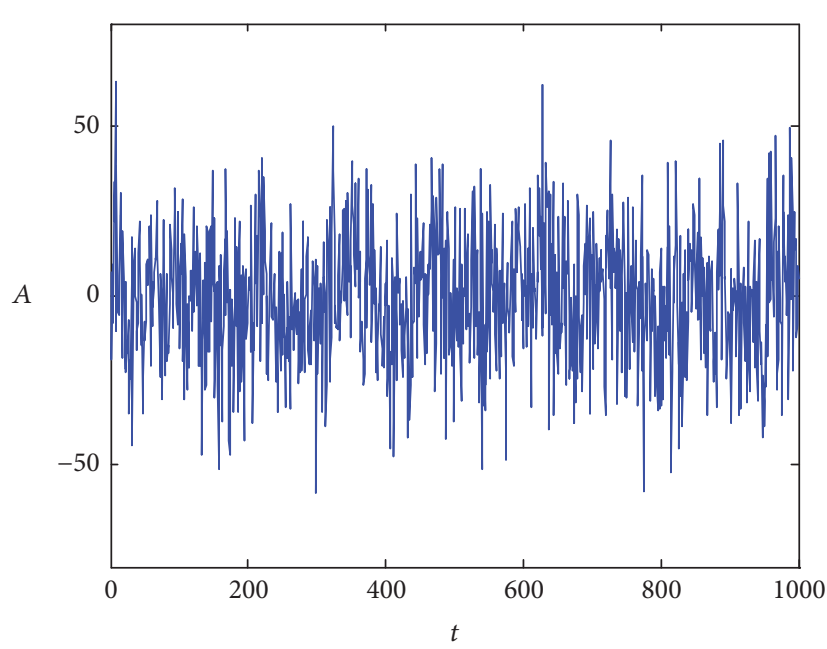

(a)

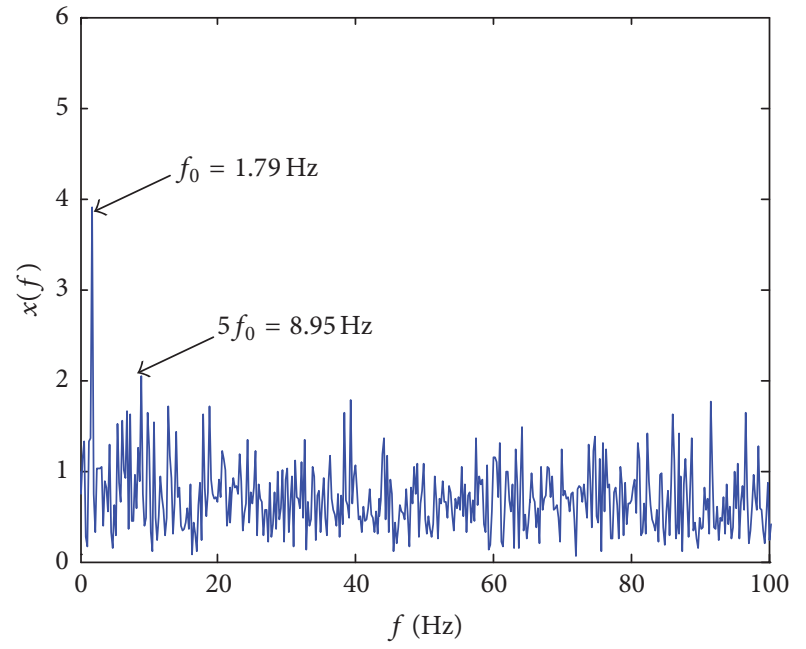

(b)

FIGURE 16: Vibration signals under the wear of floating tile: (a) time-domain waveform, (b) frequency spectrum.

elimination effect. Meanwhile, due to the principle of larger amplitude keeping in the EMD-DT and EMD-IT methods, plenty of noises with larger amplitude are not discarded in the denoising process, which makes the characteristic signals still submerge in the heavy background.

To test the effectiveness of the proposed algorithm, the same vibration signals are processed using the proposed EMD-PE method. From the feature extracting results in Figure 17, we can observe that the fault characteristics frequency of floating tile is $8.95 \mathrm{~Hz}$ and its harmonic components can be seen clearly in the spectrum, which indicates that the proposed method in this paper could successfully extract the fault features of the floating tile wear, whereas the other contrastive methods fail to detect the malfunction effectively.

Additionally, Figures 18(a) and 18(b) show the timedomain waveforms and frequency spectrum of pressure fluctuation signals under the wear of floating tile. Due to the high sediment concentration in the Yellow River, the pressure fluctuation signals suffer from serious disturbance during the signal collection and the fault feature of the floating tile cannot be clearly identified from the time-domain waveform and frequency spectrum.
Figure 19 displays the feature extracting results of pressure fluctuation signals under the wear of floating tile with the EMD-based denoising methods. Based on the observation in Figures 19(a) and 19(b), the fault characteristic information has been lost in reconstructed signals. Moreover, according to the illustration provided in Figures 19(c)-19(f), the disturbed noises during the pressure fluctuation signals collecting process are preserved by EMD-DT and EMD-IT denoising method, which is not conducive to detect the characteristic frequency signal and may lead to an incorrect diagnosis conclusion.

As shown in Figures 19(g) and 19(h), the proposed EMDPE method is utilized to process the pressure fluctuation signals for comparisons. It can be seen that the obvious peak in frequency spectrum is $8.95 \mathrm{~Hz}$, which is equal to the characteristic frequency of floating tile wear. By comparison, the frequency spectra in Figures 19(a)-19(f) with the current EMD-based denoising methods contain many complex ingredients and fault feature cannot be recognized obviously. Therefore, based on the above analysis, it is demonstrated that the proposed EMD-PE method is superior to the current EMD-based denoising methods in periodic fault signal enhancement of rotating machine. 


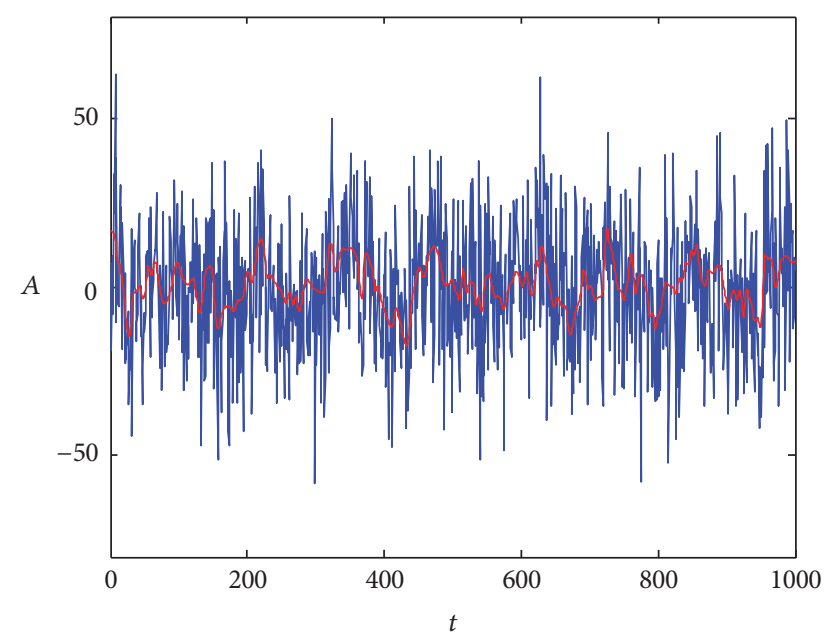

_ Original signal

(a)

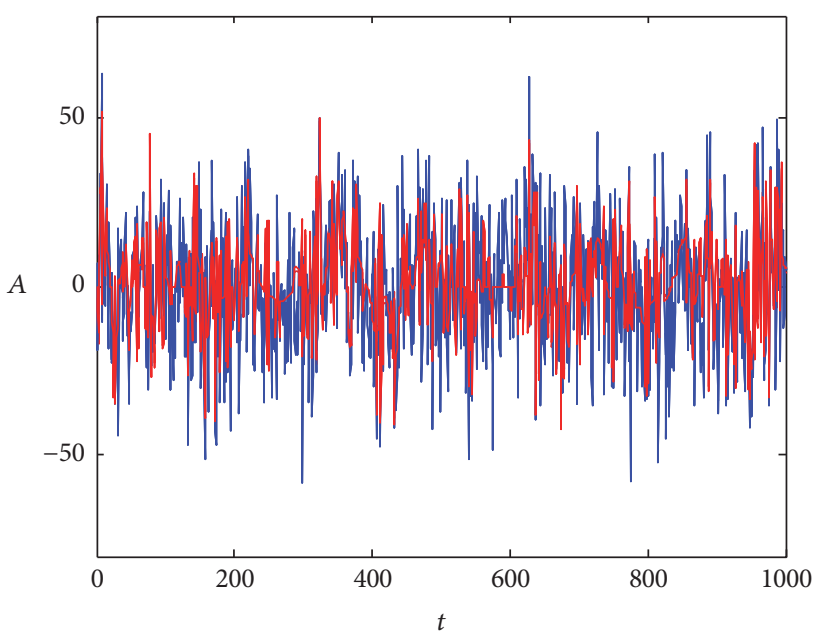

_ Original signal

— EMD-DT

(c)

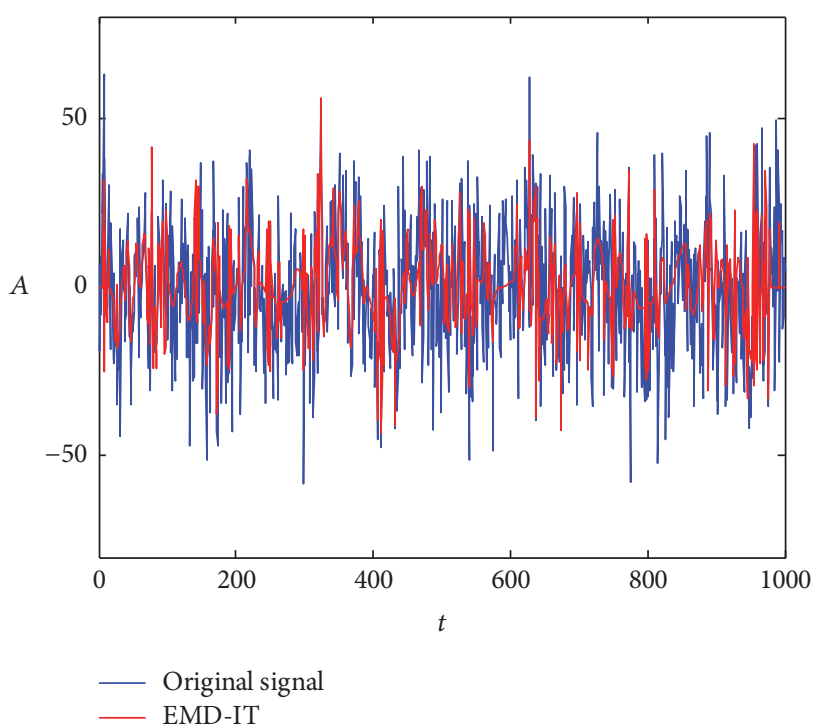

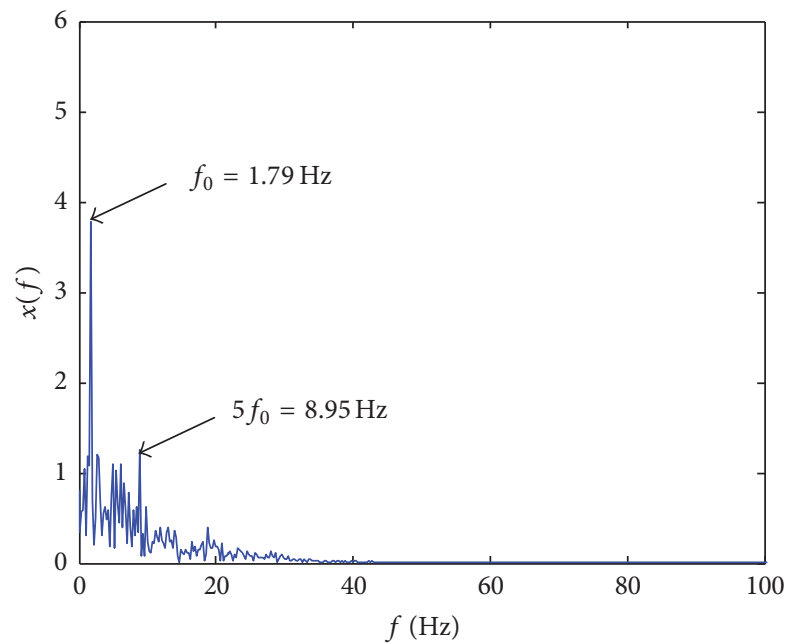

(b)

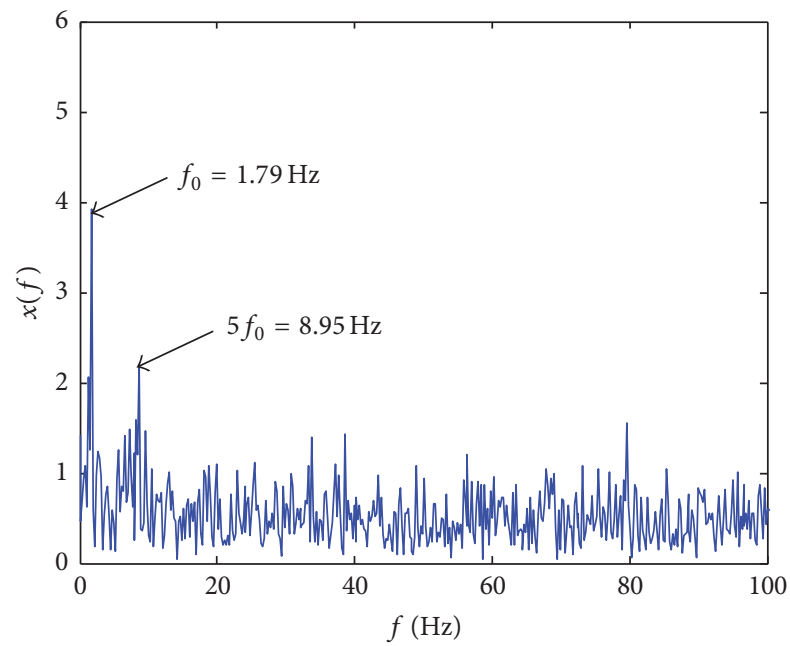

(d)

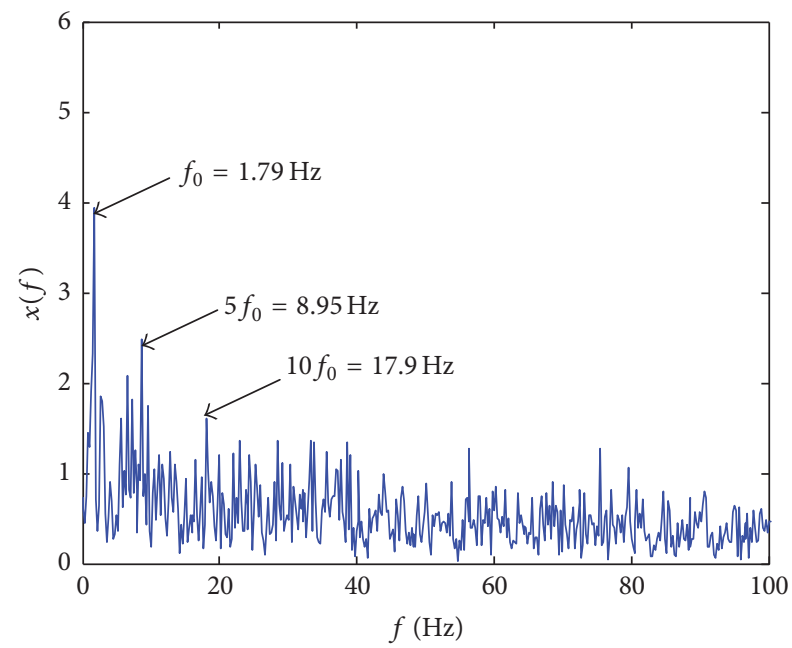




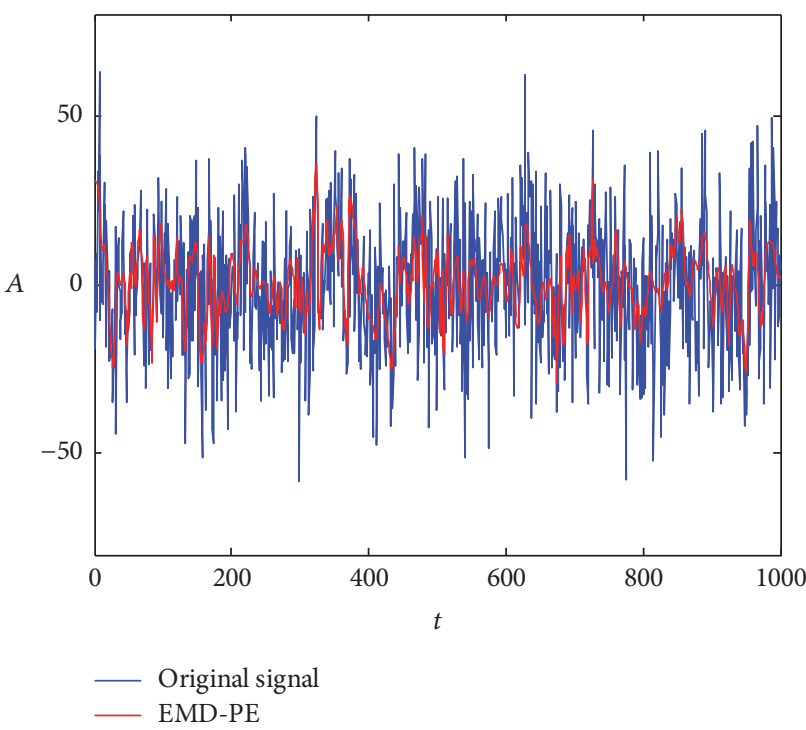

(g)

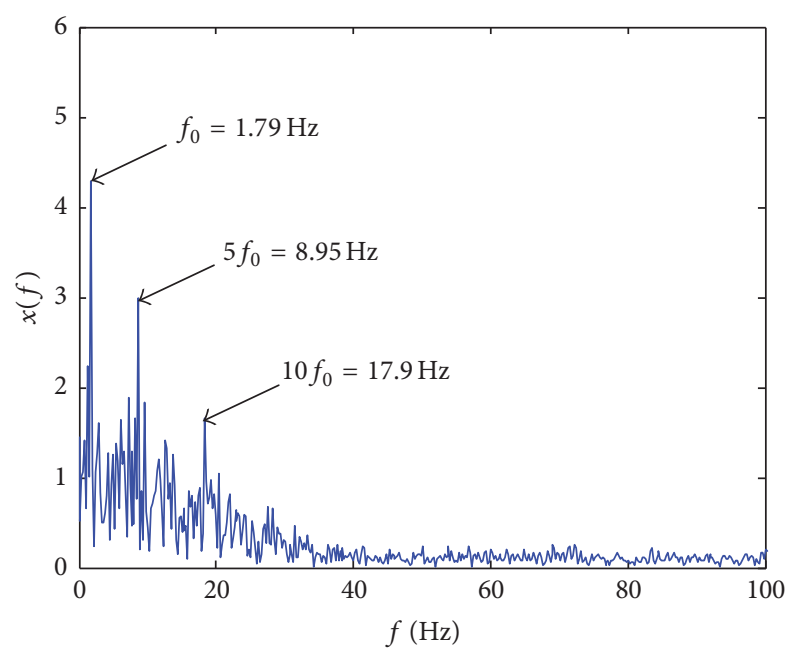

(h)

FIGURE 17: Experimental results of vibration signals under the wear of floating tile: (a) denoised signals of EMD-PR, (b) frequency spectrum of EMD-PR, (c) denoised signals of EMD-DT, (d) frequency spectrum of EMD-DT, (e) denoised signals of EMD-IT, (f) frequency spectrum of EMD-IT, (g) denoised signals of EMD-PE, and (h) frequency spectrum of EMD-PE.

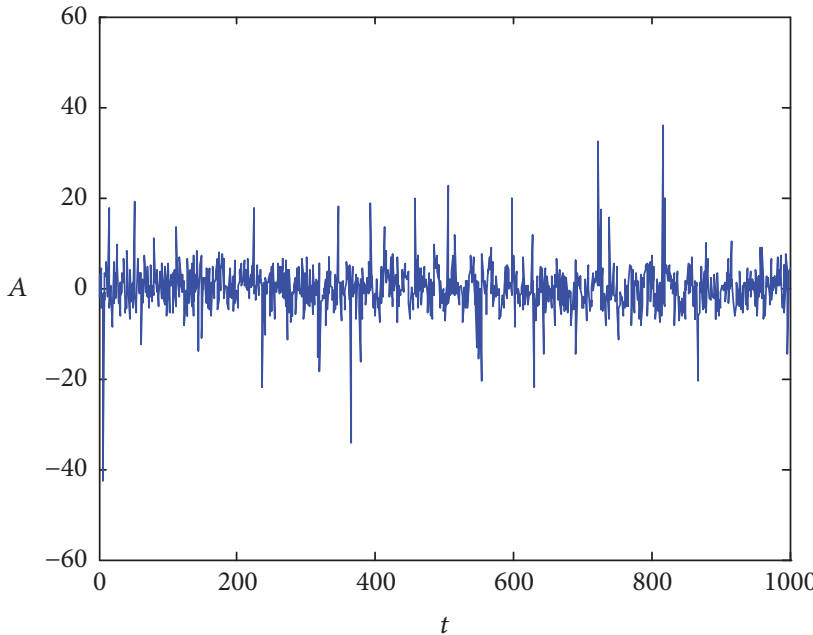

(a)

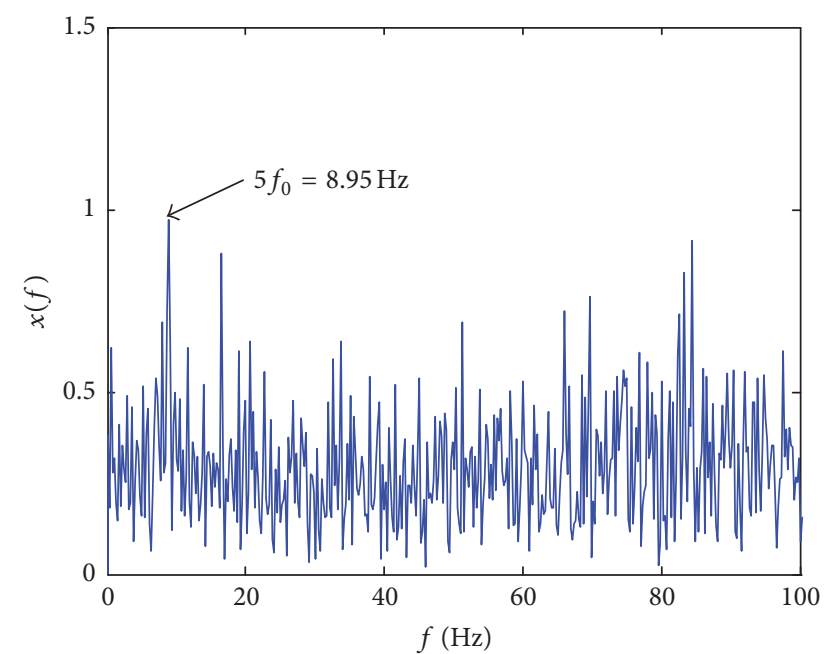

(b)

FIGURE 18: Pressure fluctuation signals under the wear of floating tile: (a) time-domain waveform, (b) frequency spectrum.

\section{Conclusion}

Considering that the early fault feature of the rotating machine is usually overwhelmed in heavy background noise, this paper proposed a probabilistic entropy EMD thresholding algorithm for periodic fault signal enhancement in rotating machine. In this method, the entropy threshold of each IMF is constructed instead of the threshold applied to $N$ sampling points of each IMF directly. The proposed novel algorithm overcomes the shortcoming of the low efficiency noise reduction effect caused by larger amplitude noise reservation and smaller feature signal reduction in the traditional denoising methods. Meanwhile, in order to make the amplitudes of all the IMF reduce in a smooth way, this paper presented a multiscale thresholding algorithm based on quantile statistics for providing probability indexes. The performance of proposed method has been evaluated under the wear of floating tile of hydroelectric turbine in upper reaches of the Yellow River. Engineering application has demonstrated that the proposed method is effective in the noise reduction and fault feature enhancement in rotating machine. 

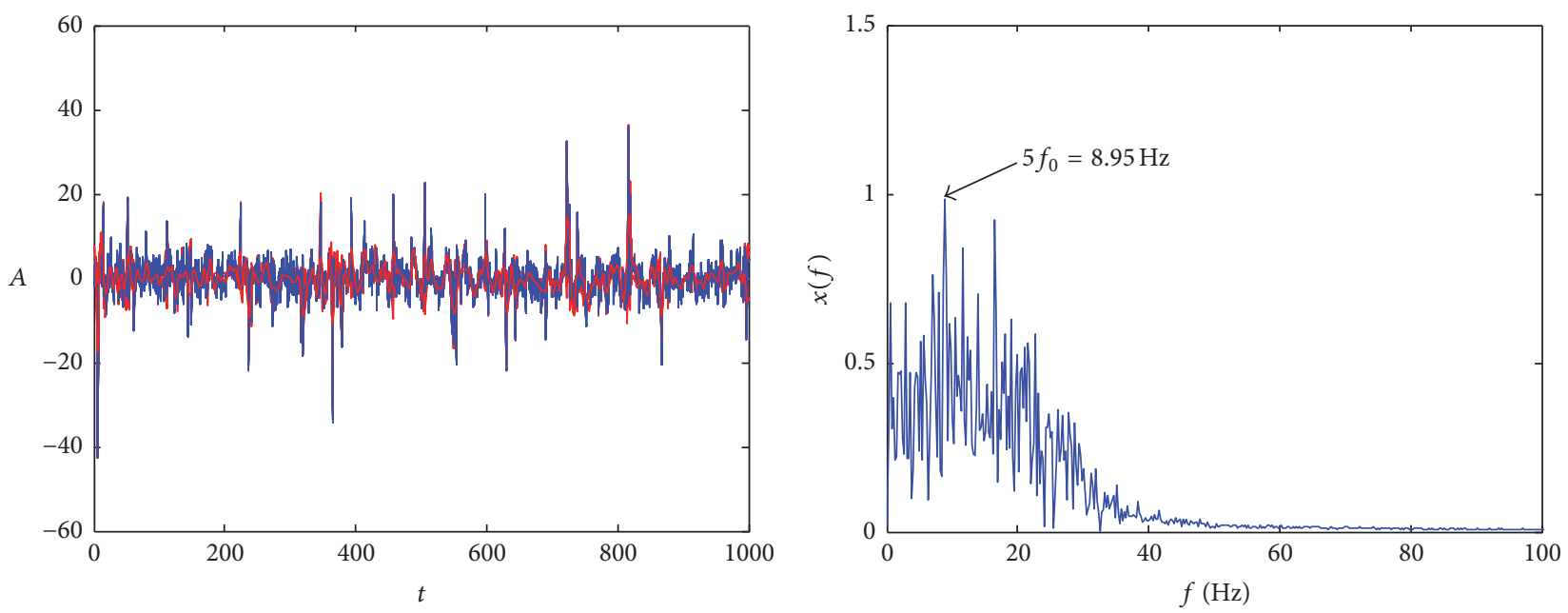

- Original signal

— EMD-PR

(a)

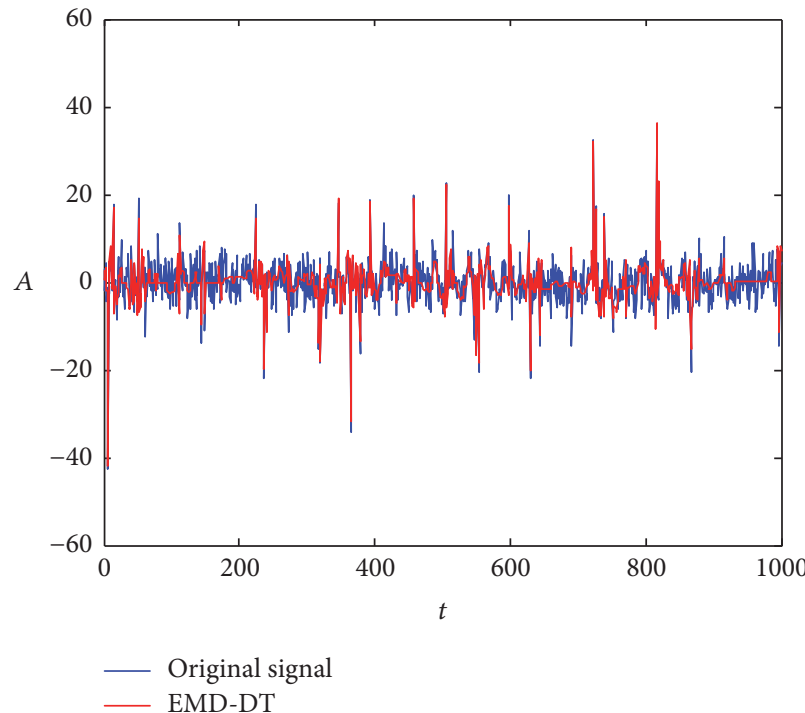

(c)

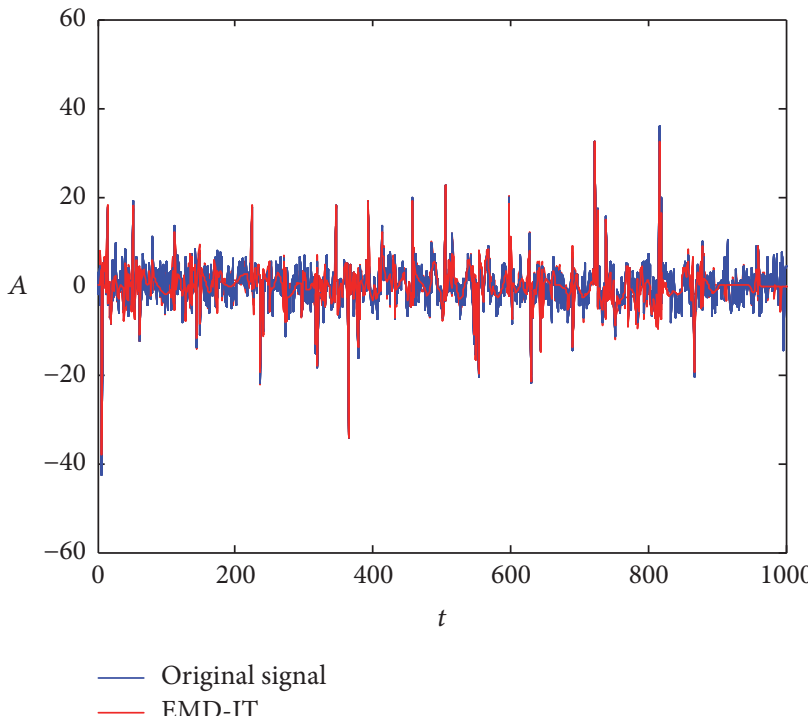

(b)

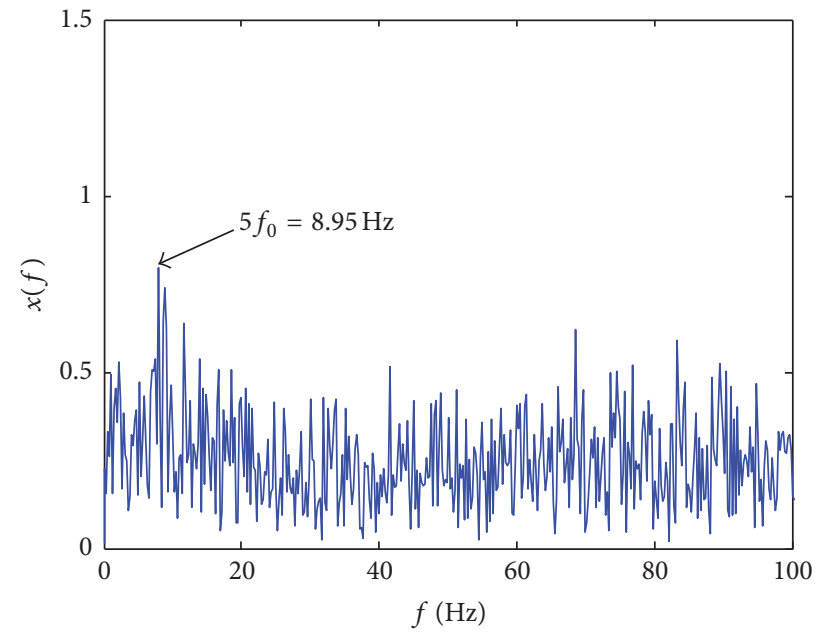

(d)

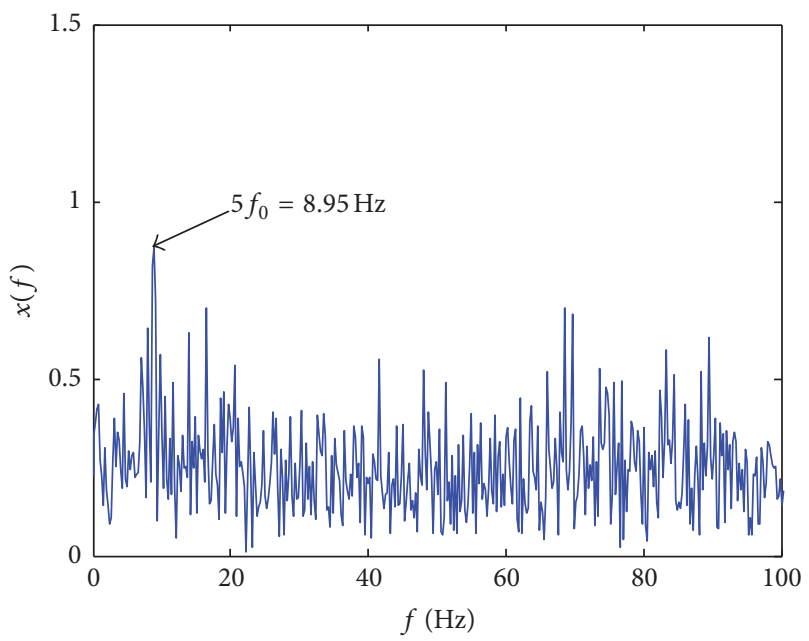




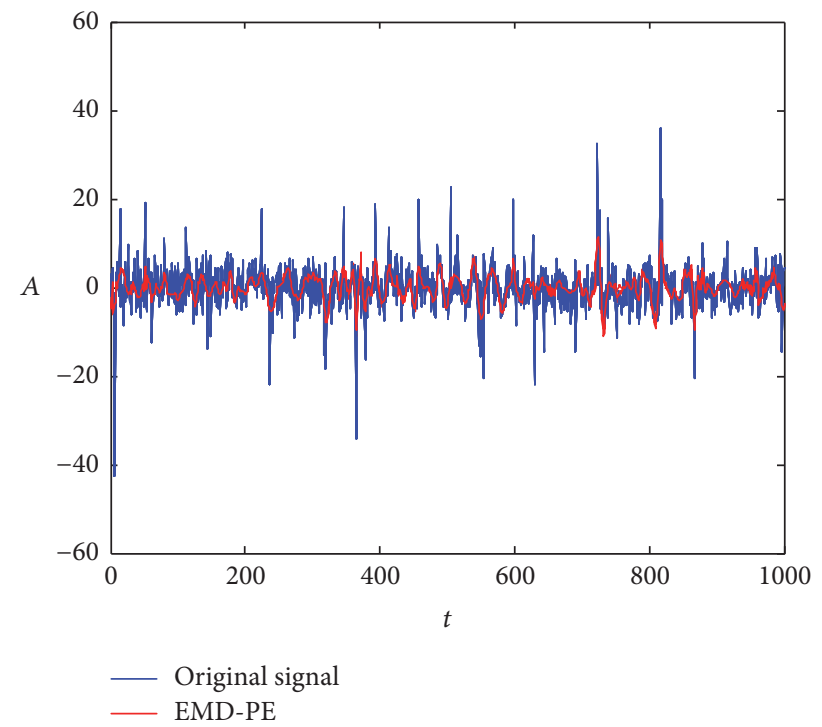

(g)

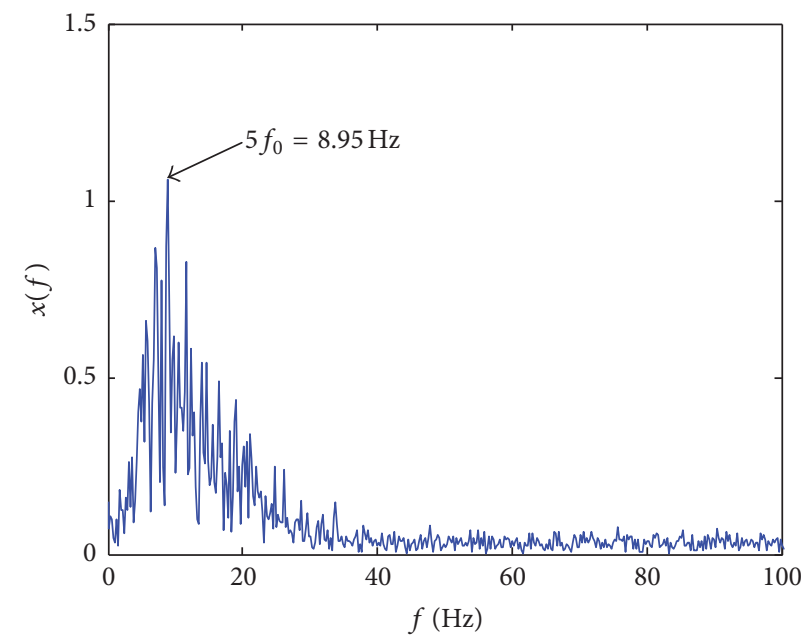

(h)

FIGURE 19: Experimental results of pressure fluctuation signals under the wear of floating tile: (a) denoised signals of EMD-PR, (b) frequency spectrum of EMD-PR, (c) denoised signals of EMD-DT, (d) frequency spectrum of EMD-DT, (e) denoised signals of EMD-IT, (f) frequency spectrum of EMD-IT, (g) denoised signals of EMD-PE, and (h) frequency spectrum of EMD-PE.

\section{Conflicts of Interest}

The authors declare that there are no conflicts of interest regarding the publication of this paper.

\section{Acknowledgments}

This research is supported by Key Program of the National Natural Science Foundation of China (Grant no. 51279161), Hydraulic Science and Technology Program of Shaanxi Province (Grant no. 2015slkj-04), and State Grid Corporation of China Research Program (522722150012).

\section{References}

[1] D. E. Bently and C. T. Hatch, Fundamentals of Rotating Machinery Diagnostics, American Society of Mechanical Engineers, New York, NY, USA, 2002.

[2] A. D. Nembhard, J. K. Sinha, and A. Yunusa-Kaltungo, "Development of a generic rotating machinery fault diagnosis approach insensitive to machine speed and support type," Journal of Sound and Vibration, vol. 337, pp. 321-341, 2015.

[3] J. Chen, Z. Li, J. Pan et al., "Wavelet transform based on inner product in fault diagnosis of rotating machinery: a review," Mechanical Systems and Signal Processing, vol. 70-71, pp. 1-35, 2016.

[4] J. Dang, R. Jia, X. Luo, H. Wu, and D. Chen, "Partly duffing oscillator stochastic resonance method and its application on mechanical fault diagnosis," Shock and Vibration, vol. 2016, Article ID 3109385, 2016.

[5] N. E. Huang, Z. Shen, S. R. Long et al., "The empirical mode decomposition and the Hilbert spectrum for nonlinear and non-stationary time series analysis," Proceedings of the Royal
Society A-Mathematical, Physical and Engineering Sciences, vol. 454, no. 1971, pp. 903-995, 1998.

[6] Y. Lei, J. Lin, Z. He, and M. J. Zuo, "A review on empirical mode decomposition in fault diagnosis of rotating machinery," Mechanical Systems and Signal Processing, vol. 35, no. 1-2, pp. 108-126, 2013.

[7] C. Mishra, A. K. Samantaray, and G. Chakraborty, "Bond graph modeling and experimental verification of a novel scheme for fault diagnosis of rolling element bearings in special operating conditions," Journal of Sound and Vibration, vol. 377, pp. 302330, 2016.

[8] X. Xue, J. Zhou, Y. Xu, W. Zhu, and C. Li, "An adaptively fast ensemble empirical mode decomposition method and its applications to rolling element bearing fault diagnosis," Mechanical Systems and Signal Processing, vol. 62, pp. 444-459, 2015.

[9] Z. H. Wu and N. E. Huang, "A study of the characteristics of white noise using the empirical mode decomposition method," Proceedings of the Royal Society A: Mathematical, Physical and Engineering Sciences, vol. 17, no. 2046, pp. 1597-1611, 2004.

[10] P. Flandrin, G. Rilling, and P. Gonçalvés, "Empirical mode decomposition as a filter bank," IEEE Signal Processing Letters, vol. 11, no. 2, pp. 112-114, 2004.

[11] B. G. Jeong, B. C. Kim, Y. H. Moon, and I. K. Eom, "Simplified noise model parameter estimation for signal-dependent noise," Signal Processing, vol. 96, pp. 266-273, 2014.

[12] P. Flandrin, P. Gonçalves, and G. Rilling, "EMD Equivalent Filter Banks, from Interpretation to Applications," in HilbertHuang Transform And Its Applications, vol. 5 of Interdisciplinary Mathematical Sciences, pp. 57-74, World Scientific, Hackensack, NJ, USA, 2005.

[13] A.-O. Boudraa and J.-C. Cexus, "EMD-based signal filtering," IEEE Transactions on Instrumentation \& Measurement, vol. 56, no. 6, pp. 2196-2202, 2007. 
[14] R. Ricci and P. Pennacchi, "Diagnostics of gear faults based on EMD and automatic selection of intrinsic mode functions," Mechanical Systems and Signal Processing, vol. 25, no. 3, pp. 821838, 2011.

[15] A.-P. Albert and A.-O. Nii, "A criterion for selecting relevant intrinsic mode functions in empirical mode decomposition," Advances in Adaptive Data Analysis, vol. 2, no. 1, pp. 1-24, 2010.

[16] O. A. Omitaomu, V. A. Protopopescu, and A. R. Ganguly, "Empirical mode decomposition technique with conditional mutual information for denoising operational sensor data," IEEE Sensors Journal, vol. 11, no. 10, pp. 2565-2575, 2011.

[17] R. Shao, W. Hu, and J. Li, "Multi-fault feature extraction and diagnosis of gear transmission system using time-frequency analysis and wavelet threshold de-noising based on EMD," Shock and Vibration, vol. 20, no. 4, pp. 763-780, 2013.

[18] G. Yang, Y. Liu, Y. Wang, and Z. Zhu, "EMD interval thresholding denoising based on similarity measure to select relevant modes," Signal Processing, vol. 109, pp. 95-109, 2015.

[19] A. Komaty, A. Boudraa, and D. Dare, "EMD-based filtering using the Hausdorff distance," in Proceedings of the 12th IEEE International Symposium on Signal Processing and Information Technology (ISSPIT '12), pp. 292-297, Ho Chi Minh City, Vietnam, December 2012.

[20] A. Komaty, A.-O. Boudraa, B. Augier, and D. Dare-Emzivat, "EMD-based filtering using similarity measure between probability density functions of IMFs," IEEE Transactions on Instrumentation and Measurement, vol. 63, no. 1, pp. 27-34, 2014.

[21] Y. Kopsinis and S. McLaughlin, "Development of EMD-based denoising methods inspired by wavelet thresholding," IEEE Transactions on Signal Processing, vol. 57, no. 4, pp. 1351-1362, 2009.

[22] D. L. Donoho and I. M. Johnstone, "Ideal spatial adaptation by wavelet shrinkage," Biometrika, vol. 81, no. 3, pp. 425-455, 1994.

[23] C.-S. Qu, T.-Z. Lu, and Y. Tan, "A modified empirical mode decomposition method with applications to signal de-noising," Acta Automatica Sinica, vol. 36, no. 1, pp. 67-73, 2010.

[24] N. R. Saggurti and J. Shankar, "EMD based clear recursive thresholding (EMD-CRT) for speech enhancement," in Proceedings of the International Conference on Signal Processing, Computing and Control, ISPCC 2015, pp. 149-154, Solan, India, September 2015.

[25] C. Bandt and B. Pompe, "Permutation entropy: a natural complexity measure for time series," Physical Review Letters, vol. 88, no. 17, Article ID 174102, 2002.

[26] F. Fu-zhou, R. Guo-qiang, Z. Li-xia et al., "Research on abnormality detection method for $\mathrm{s}$ based on emd and permutation entropy," Bearing, vol. 2, pp. 53-56, 2013.

[27] Y. Kopsinis and S. McLaughlin, "Empirical mode decomposition based soft-thresholding," in Proceedings of the 16th European Signal Processing Conference, EUSIPCO 2008, IEEE, Lausanne, Switzerland, August 2008.

[28] B. Fadlallah, B. Chen, A. Keil, and J. Príncipe, "Weightedpermutation entropy: a complexity measure for time series incorporating amplitude information," Physical Review E, vol. 87, no. 2, Article ID 022911, 2013.

[29] N. Nicolaou and J. Georgiou, "Detection of epileptic electroencephalogram based on Permutation Entropy and Support Vector Machines," Expert Systems with Applications, vol. 39, no. 1, pp. 202-209, 2012.

[30] F. C. Morabito, D. Labate, F. La Foresta, A. Bramanti, G. Morabito, and I. Palamara, "Multivariate multi-scale permutation entropy for complexity analysis of Alzheimer's disease EEG," Entropy, vol. 14, no. 7, pp. 1186-1202, 2012.

[31] J. M. Li, X. F. Chen, and Z. J. He, "Adaptive stochastic resonance method for impact signal detection based on sliding window," Mechanical Systems and Signal Processing, vol. 36, no. 2, pp. 240255, 2013. 


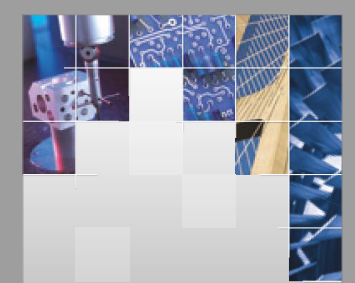

\section{Enfincering}
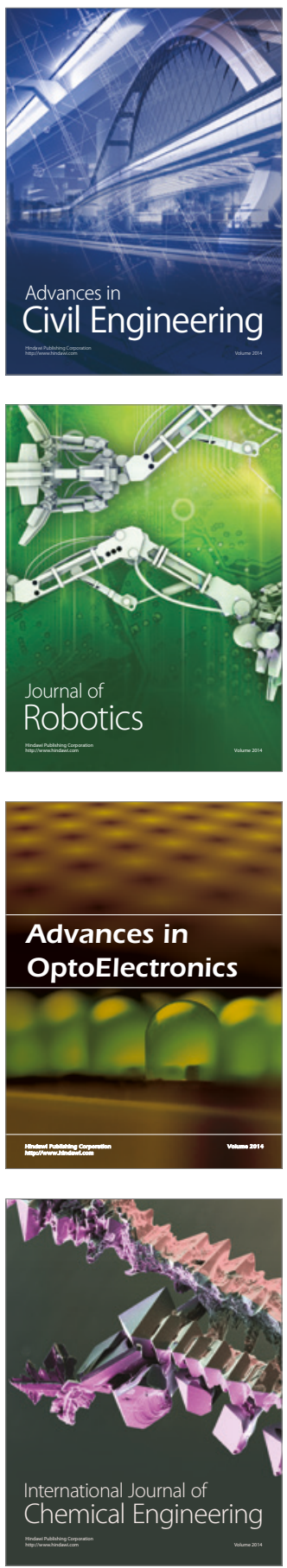

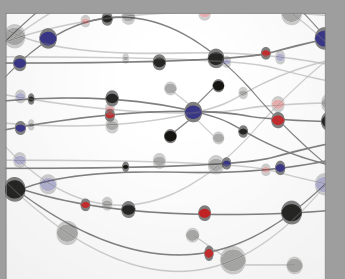

The Scientific World Journal

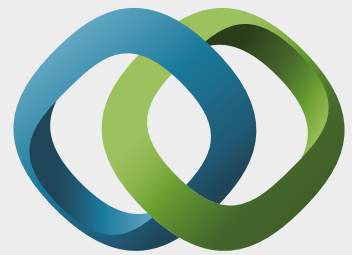

\section{Hindawi}

Submit your manuscripts at

https://www.hindawi.com
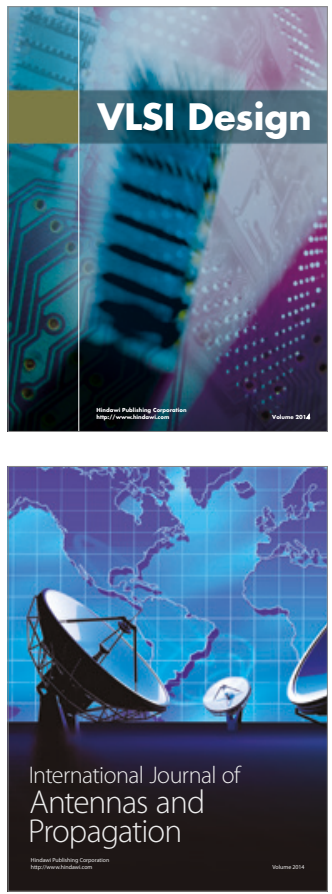

\section{Rotating}

Machinery
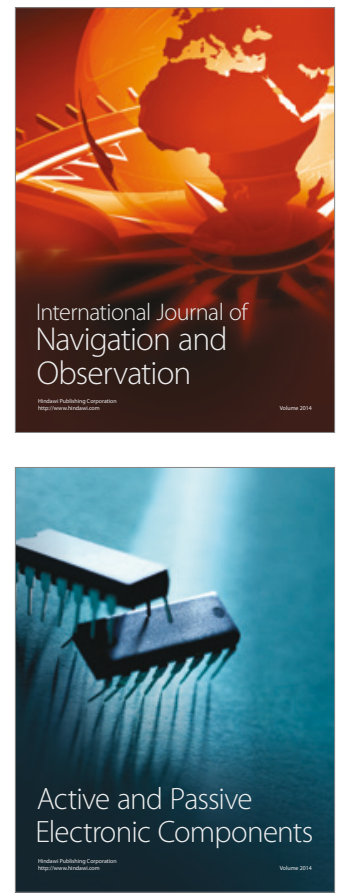
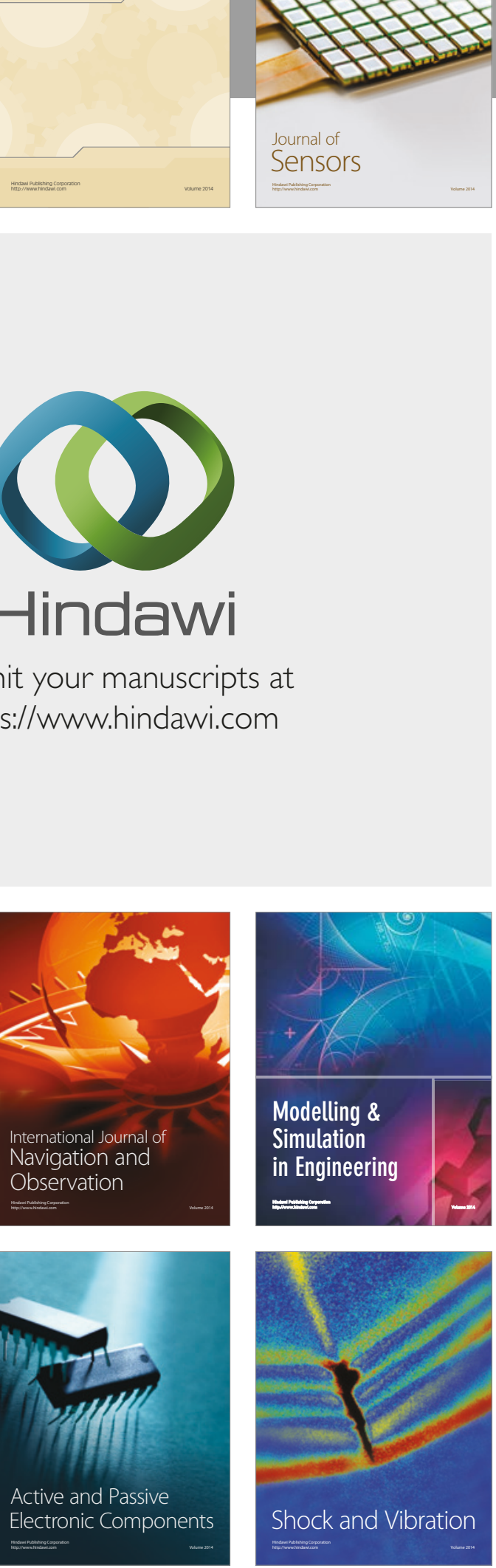
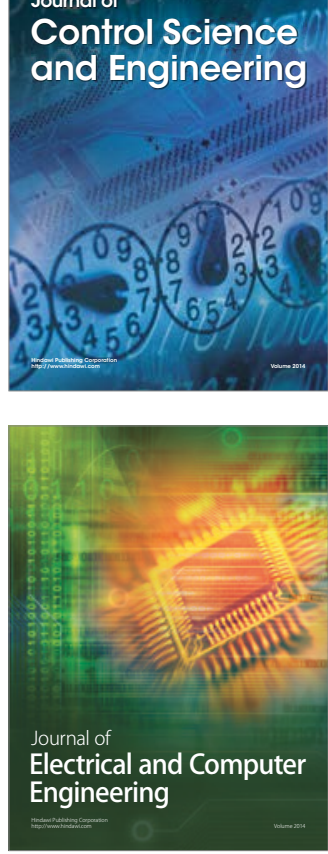

Distributed

Journal of

Control Science

and Engineering
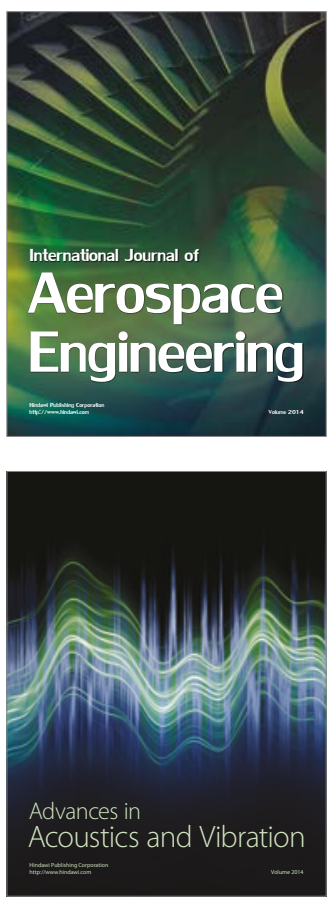

Sensor Networks 